\title{
A posteriori error estimation and adaptivity based on VMS for the incompressible Navier-Stokes equations
}

\author{
Diego Irisarri, Guillermo Hauke \\ Universidad de Zaragoza \\ Escuela de Ingeniería y Arquitectura, Área de Mecánica de Fluidos \\ C/María de Luna 3, 50018 Zaragoza, Spain \\ e-mail: dirisarri@unizar.es
}

\begin{abstract}
In this work an explicit a posteriori error estimator for the steady incompressible Navier-Stokes equations is investigated. The error estimator is based on the variational multiscale theory, where the numerical solution is decomposed in resolved scales (FEM solution) and unresolved scales (FEM error). The error is estimated locally considering the residuals that emerge from the numerical solution and the error inverse-velocity scales, $\tau$ 's, associated with each type of residual. These error scales are provided in this paper, which have been computed a-priori solving a set of local problems with unit residuals. Therefore, the computational effort to predict the error is small and its implementation in any FEM code is simple. As an application, a strategy to develop adaptive meshes with the aim of optimizing the computational effort is shown. Numerical examples are presented to test the behavior of the error estimator.

Keywords: A posteriori error estimation, adaptivity, finite elements, variational multiscale method, Navier-Stokes equations
\end{abstract}

\section{Introduction}

In the last decades, numerical methods have played an important role in fluid mechanics. Particularly, the application of the finite element method (FEM) by scientist and engineers has experienced a great progress in the field of fluid me-

5 chanics. The solution given by the FEM, as for other methods, has an inherent 
error, that is convenient to estimate in order to assess the quality of the numerical solution. For this reason, a posteriori error estimators have become a useful and necessary tool for FEM users [3, 9]. Furthermore, a posteriori error estimators can be employed to optimize the computational resources driving adaptive meshes that concentrate the elements in the regions of the domain where the error is greater. In this work, an error estimator is applied to the incompressible Navier-Stokes equations.

The present a posteriori error estimator is developed within the variational multiscale framework (VMS). Basically, the VMS theory consists in splitting 15 both the trial and test functions into coarse scales, related to the numerical solution, and fine scales, connected with the unresolved scales, [47, 49]. This point of departure has been widely used in many different areas of numerical methods such as for developing stabilized methods [51, 24, 35, 61, 4, 36, 72], for modeling small scales in LES turbulence models [14, 24, 1, 71], and for 20 estimating the numerical error. Application of the latter can be found in many applications, like fluid mechanics $[39,40,42,41,43,38,37,54,53,55,6,5,68,73$, $33,12,13,64]$, elliptic problems [58, 59, 52], and elasticity [63, 45, 8]. A recent application of VMS error estimation to the propagation of error in uncertainty quantification has been published in [25]. In this paper, previous technology developed for the elliptic problems, the transport equation, Stokes flow and the compressible Navier-Stokes equations is extended to the incompressible NavierStokes equations.

The incompressible Navier-Stokes equations present some particularities from the numerical point of view. On one hand, the equations are nonlinear due to 30 the convective term, requiring more complex solvers than for linear problems, such as the Stokes equations. Also, when the convective term is dominant, spurious oscillations can appear if the FEM formulation is not treated properly. Finally, due to the incompressibility constraint, a saddle point problem must be handled to solve these equations. As it is well known, the approximations 35 for the velocity and pressure spaces must satisfy the Babuška-Brezzi condition. These difficulties can be extended to the a posteriori error estimator techniques 
since the error is governed by the dual problem. In this manuscript, the numerical solution has been obtained using a stabilized FEM following the work of Franca et al. [29]. This formulation enables to circumvent the Babuška-Brezzi 40 condition, and thus, the same approximation functions are selected for the velocities and the pressure. Linear and bilinear shape functions are employed for triangles and quadrilaterals, respectively.

Many authors have drawn their attention to a posteriori error estimation applied to the Stokes equations and the incompressible Navier-Stokes equations.

45 In general, there are similarities in the procedure to tackle the error estimation in both equations, although the latter is more demanding due to the convective term. In fact, usually the Stokes equations are considered as the previous benchmark to test the error estimators before addressing the Navier-Stokes equations.

For the Stokes equations, we remark the work of Verfürth [76], Kay and 50 Silvester [56], Bank and Welfert [11] and, more recently, Larsson et al. [60] that propose implicit estimators which imply the solution of local problems at the element level. The error is measured in energy-like norms. On the other hand, Ainsworth and Oden [2] and Nobile [65], develop implicit error estimators which provide the lower and upper bounds of the error. In [74], Russo proposes an ${ }_{55}$ explicit error estimator for the MINI-element employing bubble functions. Song et al. [75] take advantage of the VMS to develop an error estimator oriented to generate adapted meshes. As regards the Navier-Stokes equations, Johnson and coworkers established residual-based error estimates in various norms and their application to incompressible flow can be found in [46]. Ainsworth and Oden

${ }_{60}$ in $[67,2]$ established an error estimation measured in a energy-like norm which is bounded. Berrone presented a residual-based approach in $[17,16]$, where the influence of the Reynolds number is made explicit and the error estimate is used to adapt the mesh. As for error estimators based on VMS, Zheng et al. [77] developed a simple error estimator based on a local projection which is used for ${ }_{65}$ driving adaptive meshes. In the field of finite volume methods, Colomés et al. [26] develop an explicit VMS error estimator where the fine scales are modeled by the flow subgrid time-scales. 
In this paper, a residual-based a posteriori error estimator is presented for the Navier-Stokes equation. The way of linking the residuals with the error 70 is carried out via the variational multiscale theory (VMS). Recall that VMS consists in splitting both the trial and test functions into coarse scales, related to the numerical solution, and fine scales, connected with the unresolved scales, $[47,49]$. This decomposition allows the study of the interaction of the coarse scales into the fine scales, and therefore, this theory has been widely employed to develop stabilized methods and to estimate the committed error in numerical methods, as mentioned above.

The explicit estimator predicts the error by multiplying the residuals with the corresponding error time-scales (or inverse-velocity scales in the case of $H^{1}$ seminorms), $\tau$ 's. These $\tau$ 's, which are computed a-priori, model the effect of the residuals on the fine scales. Futhermore, explicit error estimators need little computational cost since a simple post-processing of the residuals leads to the estimation, although the accuracy in the estimation can be reduced. In this work, we treat the problem as a saddle point problem, considering the pressure a Lagrange multiplier and, therefore, focusing the efforts on the analysis of the 85 error in the velocity field. The error estimation is analyzed in problems that reach the steady state; thus, turbulent flows at high Reynolds numbers are not handled.

The paper is organized as follows: in Section 2, we present the NavierStokes problem and the stabilized finite element method that is employed. Also, the variational multiscale theory is established, which decomposes the scales of the problem and is exploited to estimate the error in this work. Section 3 is devoted to explain the explicit error estimator that is developed. The adaptive mesh refinement strategy based on the error estimator is described in Section 4. Several numerical examples are shown in Section 5. Local and global error estimations are presented. Finally, conclusions of this work are drawn in Section6. 


\section{The incompressible Navier-Stokes equations}

Let $\Omega$ be a domain in $\mathbb{R}^{n_{\mathrm{sd}}}$ with boundary $\Gamma$, where $n_{\mathrm{sd}}$ is the number of spatial dimensions of the problem. In this case $n_{\mathrm{sd}}=2$. According to the boundary conditions, the boundary is partitioned in two parts: $\Gamma_{g}$, where Dirichlet boundary conditions are imposed and $\Gamma_{h}$, where Neumann boundary condition are defined, such that $\Gamma_{g} \cup \Gamma_{h}=\Gamma$ and $\Gamma_{g} \cap \Gamma_{h}=\emptyset$. The steady incompressible Navier-Stokes equations read

$$
\left\{\begin{array}{rlll}
\boldsymbol{u} \cdot \nabla \boldsymbol{u}+\nabla p-\nu \nabla^{2} \boldsymbol{u} & =\boldsymbol{f} & \text { in } & \Omega \\
\nabla \cdot \boldsymbol{u} & =\mathbf{0} & \text { in } & \Omega \\
\boldsymbol{u} & =\mathrm{g} & \text { on } & \Gamma_{g} \\
\mathcal{B} \boldsymbol{u}=\nu \nabla \boldsymbol{u} \cdot \boldsymbol{n} & =\boldsymbol{h} & \text { on } & \Gamma_{h}
\end{array}\right.
$$

where $\boldsymbol{u}=(u, v)$ and $p$ are the unknown variables. $\boldsymbol{u}$ represents the velocity vector and $\nu$ the kinematic viscosity, which is assumed constant. In this work, we call $p$ the pressure although, in fact, $p=\frac{p_{\text {mech }}}{\rho}$ where $p_{\text {mech }}$ is the mechanical pressure and $\rho$ is the density, that is considered constant. Finally, $\mathbf{g}=\left(g_{x}, g_{y}\right)^{T}$ and $\boldsymbol{h}=\left(h_{x}, h_{y}\right)^{T}$ are the Dirichlet and Neumann boundary conditions, respectively. When the viscosity is constant, the viscous term can be written as a Laplacian. In this simpler form, the above natural condition possesses a physical meaning, namely, the variation of the velocity in the outward normal direction of the boundary. In doing so, we avoid boundary conditions on pseudo-tractions, which lack physical meaning (see for instance [50, 28]).

In short, problem (1) can be expressed as

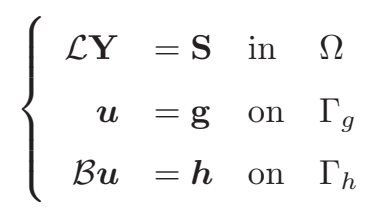

where $\mathcal{L}$ is the equation differential operator, $\mathcal{B}$ is the differential operator which 115 acts on the Neumann boundaries and arises from integration by parts. $\mathbf{Y}=$ $(u, p, v)^{T}$ is the unknown vector and $\mathbf{S}=\left(f_{x}, 0, f_{y}\right)^{T}$ represents the source term. 
The variational form is obtained multiplying the strong form by weighting functions and integrating by parts. To set it up we need to introduce the velocity weighting and trial solution spaces, $\mathcal{V}$ and $\mathcal{S}$, and the pressure weighting and trial spaces, $\mathcal{Q}$ and $\mathcal{P}$. Indeed,

$$
\begin{aligned}
& \mathcal{V}=\left\{\boldsymbol{v} \in\left(H^{1}(\Omega)^{n_{\mathrm{sd}}}\right) \mid \boldsymbol{v}=\mathbf{0} \text { on } \Gamma_{g}\right\} \\
& \mathcal{S}=\left\{\boldsymbol{u} \in\left(H^{1}(\Omega)^{n_{\mathrm{sd}}}\right) \mid \boldsymbol{u}=\mathbf{g} \text { on } \Gamma_{g}\right\} \\
& \mathcal{Q}=\left\{q \in L^{2}(\Omega) \cap H^{1}(\Omega) \text { s.t. } \quad \int_{\Omega} q=0\right\} \\
& \mathcal{P}=\left\{p \in L^{2}(\Omega) \cap H^{1}(\Omega) \text { s.t. } \quad \int_{\Omega} p=0\right\}
\end{aligned}
$$

The variational form can be written as: Find $\{\boldsymbol{u}, p\} \in \mathcal{S} \times \mathcal{P}$ such that

$$
B(\boldsymbol{u}, p ; \boldsymbol{v}, q)=F(\boldsymbol{v}, q), \forall\{\boldsymbol{v}, q\} \in \mathcal{V} \times \mathcal{Q}
$$

with

$$
B(\boldsymbol{u}, p ; \boldsymbol{v}, q)=(\boldsymbol{u} \cdot \nabla \boldsymbol{u}, \boldsymbol{v})+(\nabla p, \boldsymbol{v})+\nu(\nabla \boldsymbol{u}, \nabla \boldsymbol{v})-(\nabla \cdot \boldsymbol{u}, q)
$$

and

$$
F(\boldsymbol{v}, q)=(\boldsymbol{v}, \boldsymbol{f})+(\boldsymbol{v}, \boldsymbol{h})_{\Gamma_{h}}
$$

Note that in order to obtain the above natural boundary condition, the pressure term has not been integrated by parts.

Now, in order to establish the FEM formulation, we select finite dimensional spaces. Let $\mathcal{S}_{h} \subset \mathcal{S}$ and $\mathcal{V}_{h} \subset \mathcal{V}$ be the trial and weighting finite dimensional spaces for the velocity. Similarly, we define $\mathcal{P}_{h} \subset \mathcal{P}$ and $\mathcal{Q}_{h} \subset \mathcal{Q}$ as the trial and weighting finite dimensional spaces for the pressure. This spaces represent a partition $\mathscr{C}_{h}$ formed by elements $\Omega^{e}$.

The Galerkin method is set as: Find $\left\{\boldsymbol{u}_{h}, p_{h}\right\} \in \mathcal{S}_{h} \times \mathcal{P}_{h}$ such that

$$
B\left(\boldsymbol{u}_{h}, p_{h} ; \boldsymbol{v}_{h}, q_{h}\right)=F\left(\boldsymbol{v}_{h}, q_{h}\right), \quad \forall\left\{\boldsymbol{v}_{h}, q_{h}\right\} \in \mathcal{V}_{h} \times \mathcal{P}_{h}
$$

with

$$
B\left(\boldsymbol{u}_{h}, p_{h} ; \boldsymbol{v}_{h}, q_{h}\right)=\left(\boldsymbol{u}_{h} \cdot \nabla \boldsymbol{u}_{h}, \boldsymbol{v}_{h}\right)+\left(\nabla p_{h}, \boldsymbol{v}_{h}\right)+\nu\left(\nabla \boldsymbol{u}_{h}, \nabla \boldsymbol{v}_{h}\right)-\left(\nabla \cdot \boldsymbol{u}_{h}, q_{h}\right)
$$


and

$$
F\left(\boldsymbol{v}_{h}, q_{h}\right)=\left(\boldsymbol{v}_{h}, \boldsymbol{f}\right)+\left(\boldsymbol{v}_{h}, \boldsymbol{h}\right)_{\Gamma_{h}}
$$

\subsection{Stabilized method}

In order to obtain stable solutions, it is well known that the finite element spaces for velocity and pressure must satisfy the Babuška-Brezzi or inf-sup condition $[19,7]$. A way of circumventing this condition is to introduce stabilization terms in the discrete formulation. Besides, additional stabilized terms must be included in the FEM formulation since spurious oscillations can appear in the velocity field for convection-dominated regimes. Many authors have developed stabilized formulations for the Stokes problem [20, 50, 70, 31, 30, 57] and NavierStokes [15, 27, 62, 23]. Following the paper [29], weighted residuals are added in the FEM formulation.

In this work, the solution is driven towards steady state through a transient. Thus, the unsteady stabilized method reads: Find $\left\{\boldsymbol{u}_{h}, p_{h}\right\} \in \mathcal{S}_{h} \times \mathcal{P}_{h}$ such that

$$
B_{\text {stab }}\left(\boldsymbol{u}_{h}, p_{h} ; \boldsymbol{v}_{h}, q_{h}\right)=F_{\text {stab }}\left(\boldsymbol{v}_{h}, q_{h}\right), \quad \forall\left\{\boldsymbol{v}_{h}, q_{h}\right\} \in \mathcal{V}_{h} \times \mathcal{P}_{h}
$$

with

$$
\begin{aligned}
B_{\text {stab }}\left(\boldsymbol{u}_{h}, p_{h} ; \boldsymbol{v}_{h}, q_{h}\right)= & \left(\frac{\partial \boldsymbol{u}_{h}}{\partial t}, \boldsymbol{v}_{h}\right)+\left(\boldsymbol{u}_{h} \cdot \nabla \boldsymbol{u}_{h}, \boldsymbol{v}_{h}\right)+\left(\nabla p_{h}, \boldsymbol{v}_{h}\right)+ \\
& \nu\left(\nabla \boldsymbol{u}_{h}, \nabla \boldsymbol{v}_{h}\right)-\left(\nabla \cdot \boldsymbol{u}_{h}, q_{h}\right)+ \\
& \sum_{\Omega^{e} \in \mathscr{C}_{h}} \tau_{\operatorname{mom}}\left(\frac{\partial \boldsymbol{u}_{h}}{\partial t}+\boldsymbol{u}_{h} \cdot \nabla \boldsymbol{u}_{h}+\nabla p_{h}-\nu \Delta \boldsymbol{u}_{h}, \boldsymbol{u}_{h} \cdot \nabla \boldsymbol{v}_{h}\right. \\
& \left.\left.+\nabla q_{h}-\nu \Delta \boldsymbol{v}_{h}\right)\right)_{\Omega^{e}}+\delta\left(\nabla \cdot \boldsymbol{u}_{h}, \nabla \cdot \boldsymbol{v}_{h}\right)
\end{aligned}
$$

and

$$
\left.F_{\text {stab }}\left(\boldsymbol{v}_{h}, q_{h}\right)=\left(\boldsymbol{v}_{h}, \boldsymbol{f}\right)+\left(\boldsymbol{v}_{h}, \boldsymbol{h}\right)_{\Gamma_{h}}+\sum_{\Omega^{e} \in \mathscr{C}_{h}} \tau_{\mathrm{mom}}\left(\boldsymbol{f}, \boldsymbol{u}_{h} \cdot \nabla \boldsymbol{v}_{h}+\nabla q_{h}-\nu \Delta \boldsymbol{v}_{h}\right)\right)_{\Omega^{e}}
$$

where $\delta$ and $\tau_{\text {mom }}$ are stablity parameters. They are taken from Codina [23]

$$
\tau_{\mathrm{mom}}=\left(\frac{c_{1} \nu}{h^{2}}+\frac{c_{2}|u|}{h}\right)^{-1} \quad \delta=\frac{c_{3} h^{2}}{\tau_{\mathrm{mom}}}
$$


The constants $c_{1}, c_{2}$ and $c_{3}$ are taken as $c_{1}=4, c_{2}=2$ and $c_{3}=1$. Note that other definitions exist that use the metric of the mesh, such as $[44,14]$.

This stabilized formulation allows us to employ the same shape functions for the velocity and the pressure. Particularly, we select linear elements for triangles and bilinear elements for quadrilaterals.

\subsection{The variational multiscale background}

In the VMS framework, both the trial and test function spaces are decomposed into the resolved and unresolved subsets, $\mathcal{S}=\overline{\mathcal{S}} \oplus \mathcal{S}^{\prime}$ and $\mathcal{V}=\overline{\mathcal{V}} \oplus \mathcal{V}^{\prime}$ Due to the multiscale decomposition, the variables are divided into two parts such that

$$
\begin{array}{ll}
\mathbf{Y}=\overline{\mathbf{Y}}+\mathbf{Y}^{\prime} & \overline{\mathbf{Y}} \in \overline{\mathcal{S}}, \quad \mathbf{Y}^{\prime} \in \mathcal{S}^{\prime} \\
\boldsymbol{W}=\overline{\boldsymbol{W}}+\boldsymbol{W}^{\prime} & \overline{\boldsymbol{W}} \in \overline{\mathcal{V}}, \quad \boldsymbol{W}^{\prime} \in \mathcal{V}^{\prime}
\end{array}
$$

Thus, the variational formulation can be split into

$$
\begin{gathered}
B(\overline{\boldsymbol{u}}, \bar{p} ; \overline{\boldsymbol{v}}, \bar{q})+B\left(\boldsymbol{u}^{\prime}, p^{\prime} ; \overline{\boldsymbol{v}}, \bar{q}\right)=F(\overline{\boldsymbol{v}}, \bar{q}), \forall\{\overline{\boldsymbol{v}}, \bar{q}\} \in \overline{\mathcal{V}} \times \overline{\mathcal{Q}} \\
B\left(\overline{\boldsymbol{u}}, \bar{p} ; \boldsymbol{v}^{\prime}, q^{\prime}\right)+B\left(\boldsymbol{u}^{\prime}, p^{\prime} ; \boldsymbol{v}^{\prime}, q^{\prime}\right)=F\left(\boldsymbol{v}^{\prime}, q^{\prime}\right), \quad \forall\left\{\boldsymbol{v}^{\prime}, q^{\prime}\right\} \in \mathcal{V}^{\prime} \times \mathcal{Q}^{\prime}
\end{gathered}
$$

Remark 1. Due to the decomposition, the convective term is split into the following terms,

$$
\boldsymbol{u} \cdot \nabla \boldsymbol{u}=\overline{\boldsymbol{u}} \cdot \nabla \overline{\boldsymbol{u}}+\overline{\boldsymbol{u}} \cdot \nabla \boldsymbol{u}^{\prime}+\boldsymbol{u}^{\prime} \cdot \nabla \overline{\boldsymbol{u}}+\boldsymbol{u}^{\prime} \cdot \nabla \boldsymbol{u}^{\prime}
$$

The first term on the RHS of equation corresponds to the coarse scales whereas the error terms are involved in the last three terms. The last and the second-to-last terms are neglected with respect to the first term on the RHS since we suppose that $\left\|\boldsymbol{u}^{\prime}\right\|<<\|\overline{\boldsymbol{u}}\|$.

Taking the variational multiscale formulation, Eq. (15), the second equation refers to the fine scales of the problem. Mainly, we focus our attention on this equation to estimate the FEM error. The fine-scale variational form can be expressed as: Find $\boldsymbol{u}^{\prime} \in \mathcal{S}^{\prime}$ and $p^{\prime} \in \mathcal{P}^{\prime}$ such that 


$$
\begin{aligned}
\left(\overline{\boldsymbol{u}} \cdot \nabla \boldsymbol{u}^{\prime}, \boldsymbol{w}^{\prime}\right)+\left(\nabla p^{\prime}, \boldsymbol{w}^{\prime}\right)+\nu\left(\nabla \boldsymbol{u}^{\prime}, \nabla \boldsymbol{w}^{\prime}\right)= & \left(\boldsymbol{f}-\overline{\boldsymbol{u}} \cdot \nabla \overline{\boldsymbol{u}}-\nabla \bar{p}+\nu \Delta \overline{\boldsymbol{u}}, \boldsymbol{w}^{\prime}\right)_{\widetilde{\Omega}} \\
& -\left(\llbracket \mathcal{B} \overline{\boldsymbol{u}} \rrbracket_{E}, \boldsymbol{w}^{\prime}\right)_{\widetilde{\Gamma}} \\
& -\left(\mathcal{B} \overline{\boldsymbol{u}}-\boldsymbol{h}, \boldsymbol{w}^{\prime}\right)_{\Gamma_{h}} \quad \forall \boldsymbol{w}^{\prime} \in \mathcal{V}^{\prime}
\end{aligned}
$$

$$
\left(\nabla \cdot \boldsymbol{u}^{\prime}, q^{\prime}\right)=\left(-\nabla \cdot \overline{\boldsymbol{u}}, q^{\prime}\right) \forall q^{\prime} \in \mathcal{P}^{\prime}
$$

where $\widetilde{\Omega}$ is the union of the element interiors and $\widetilde{\Gamma}$ is the internal element boundaries, i.e., $\widetilde{\Gamma}=\cup E \backslash \Gamma$, with $E$ being the edges of the partition $\mathscr{C}_{h}$. Also, $\llbracket \cdot \rrbracket_{E}$ denotes the jump operator that takes into account the derivative discontinuities of $\frac{\partial \overline{\boldsymbol{u}}}{\partial \boldsymbol{n}}$ across the element edges. For a velocity field $\boldsymbol{u}=(u, v)^{T}$, and an edge shared by elements $\Omega^{+}$and $\Omega^{-}$, it is defined as

$$
\llbracket \mathcal{B} \overline{\boldsymbol{u}} \rrbracket_{E}=\nu\left(\left.\nabla \bar{u}\right|_{\partial \Omega^{+} \cap E} \cdot \boldsymbol{n}^{+}+\left.\nabla \bar{u}\right|_{\partial \Omega^{-} \cap E} \cdot \boldsymbol{n}^{-},\left.\nabla \bar{v}\right|_{\partial \Omega^{+} \cap E} \cdot \boldsymbol{n}^{+}+\nabla \bar{v}_{\left.\right|_{\partial \Omega^{-} \cap E}} \cdot \boldsymbol{n}^{-}\right)^{T}
$$
tively.

On the LHS of Eqs. (17) and (18), there appear terms which only involve the fine scales. Particularly, the error projected to the fine-scale test functions. On the RHS, we have the residuals of the numerical solution projected to the same residuals, inter-element residuals and Neumann boundary condition residuals. The first residuals are related to the non satisfaction of the differential equation $\mathcal{L} \overline{\mathbf{Y}}-\boldsymbol{f}$ inside each element. It can be seen as the difference between the numerical and the exact solution once the differential operator is applied, $\mathcal{L} \overline{\mathbf{Y}}-$ $\mathcal{L} \mathbf{Y}$. The second and third residuals are assembled together because they emerge from the lack of continuity of the numerical solution on the element boundaries.

\section{Elementwise error estimation}

In this section, we present an explicit elemental error estimator for the Navier-Stokes equations. The residuals in the momentum and continuity equa- 
tions are the source terms in the system of differential equations to obtain an estimate of the error, as Eq. (17) states. This idea has been used extensively in the past and confirmed by the VMS theory in many examples in fluid mechanics and linear elasticity [39, 43, 37, 54, 53, 45, 52, 8, 12, 55], and it is specially suited when solutions are computed using stabilized methods. In this work, the strategy is extended to the incompressible Navier-Stokes equations, for which the error is measured in the velocity field. We consider the velocity field the main variable to take into account in the saddle point problem as the pressure is the variable that plays the role of a Lagrange multiplier.

In this error estimator, as the VMS theory shows, the residuals and the error 175 estimate are directly linked. The way to proceed is to obtain error time scales, $\tau$ 's, which represent an average of the fine scales on the element. Classically, the intrinsic time-scale $\tau$ 's have been identified with stabilization parameters. However, these $\tau$ 's also are linked to the subgrid scales or error of the numerical solution.

Taking the fine-scale equations (17), the local error estimation is carried out setting this problem on each element $\Omega^{e}$,

$$
\begin{cases}\left(\overline{\boldsymbol{u}} \cdot \nabla \boldsymbol{u}^{\prime}, \boldsymbol{w}^{\prime}\right)_{\Omega^{e}}+\left(\nabla p^{\prime}, \boldsymbol{w}^{\prime}\right)_{\Omega^{e}}+\nu\left(\nabla \boldsymbol{u}^{\prime}, \nabla \boldsymbol{w}^{\prime}\right)_{\Omega^{e}}= & \left(\boldsymbol{f}-\overline{\boldsymbol{u}} \cdot \nabla \overline{\boldsymbol{u}}-\nabla \bar{p}+\nu \Delta \overline{\boldsymbol{u}}, \boldsymbol{w}^{\prime}\right)_{\Omega^{e}} \\ & -\left(\llbracket \mathcal{B} \overline{\boldsymbol{u}} \rrbracket_{E}, \boldsymbol{w}^{\prime}\right)_{\widetilde{\Gamma}^{e}} \\ & -\left(\mathcal{B} \overline{\boldsymbol{u}}-\boldsymbol{h}, \boldsymbol{w}^{\prime}\right)_{\Gamma^{e} \cap \Gamma_{h}} \quad \forall \boldsymbol{w}^{\prime} \in \mathcal{V}^{\prime} \\ \left(\nabla \cdot \boldsymbol{u}^{\prime}, q^{\prime}\right)_{\Omega^{e}}=\left(-\nabla \cdot \overline{\boldsymbol{u}}, q^{\prime}\right)_{\Omega^{e}} \forall q^{\prime} \in \mathcal{P}^{\prime} & \end{cases}
$$


From Eq. (20) we can identify five kinds of residuals for an element,

$$
\begin{aligned}
& \mathcal{R}_{M x}=f_{x}-\boldsymbol{u} \cdot \nabla \bar{u}-\partial_{x} \bar{p}+\nu \Delta \bar{u} \text { on } \Omega^{e} \\
& \mathcal{R}_{M y}=f_{y}-\boldsymbol{u} \cdot \nabla \bar{v}-\partial_{y} \bar{p}+\nu \Delta \bar{v} \text { on } \Omega^{e} \\
& \mathcal{R}_{C}=\nabla \cdot \overline{\boldsymbol{u}} \text { on } \Omega^{e} \\
& \mathcal{R}_{S_{\perp}}=\nu \llbracket \nabla \overline{\boldsymbol{u}} \cdot \boldsymbol{n} \rrbracket \cdot \boldsymbol{n} \text { on } \partial E \in \Omega^{e} \\
& \mathcal{R}_{S_{\|}}=\nu \llbracket \nabla \overline{\boldsymbol{u}} \cdot \boldsymbol{n} \rrbracket \cdot \boldsymbol{n}_{\|} \text {on } \partial E \in \Omega^{e}
\end{aligned}
$$

The residuals $\mathcal{R}_{M x}, \mathcal{R}_{M y}, \mathcal{R}_{C}$ represent the internal residual for the momentum equation and continuity equation, respectively. On the other hand, the residuals $\mathcal{R}_{S_{\perp}}$ and $\mathcal{R}_{S_{\|}}$denote the inter-element residual due to the jumps of the FEM solution on the element boundaries. Particularly, $\mathcal{R}_{S_{\perp}}$ is the orthogonal component of the jump and $\mathcal{R}_{S_{\|}}$the parallel component with respect to the element edge.

Thus, extending previous works on VMS error estimation [39, 40, 42, 41, 43, $38,37,54,53,55,6,5,68,73,33,12,13,64,58,59,52,63,45,8]$, the error estimator is built as the sum products of each residual times the corresponding parameter, which will be explained later. Thus, a dimensionally consistent expression to compute the explicit error estimator in the $H^{1}$ seminorm is defined as

$$
\begin{aligned}
& \left|\boldsymbol{u}^{\prime}\right|_{H^{1}\left(\Omega^{e}\right)}:=\frac{1}{\sqrt{\left|\Omega^{e}\right|}}(\tau)_{H^{1}}^{\boldsymbol{u}_{\mathcal{R}_{M x}}} \sum_{i=1}^{n_{\text {bub }}}\left|\left(\mathcal{R}_{M x}, v_{\mathrm{bub}_{i}}\right)_{\Omega^{e}}\right|+ \\
& +\frac{1}{\sqrt{\left|\Omega^{e}\right|}}(\tau)_{H^{1}}^{\boldsymbol{u}_{\mathcal{R}_{M y}}} \sum_{i=1}^{n_{\text {bub }}}\left|\left(\mathcal{R}_{M y}, v_{\text {bub }_{i}}\right)_{\Omega^{e}}\right|+ \\
& +\frac{1}{\sqrt{\left|\Omega^{e}\right|}}(\tau)_{H^{1}}^{\boldsymbol{u}_{\mathcal{R}_{C}}} \sum_{i=1}^{n_{\text {bub }}}\left|\left(\mathcal{R}_{C}, v_{\mathrm{bub}_{i}}\right)_{\Omega^{e}}\right|+ \\
& +\quad \sqrt{\left|\Omega^{e}\right|}(\tau)_{H^{1}}^{\boldsymbol{u}_{\mathcal{R}_{\perp}}} \sum_{i=1}^{n_{\text {edge }}} \frac{1}{l_{i}}\left|\left(\mathcal{R}_{S_{\perp}}, v_{\text {bub }_{i}}\right)_{\Gamma^{e}}\right|+ \\
& +\sqrt{\left|\Omega^{e}\right|}(\tau)_{H^{1}}^{\boldsymbol{u}_{\mathcal{R}_{S}}} \sum_{i=1}^{n_{\text {edge }}} \frac{1}{l_{i}}\left|\left(\mathcal{R}_{S_{\|}}, v_{\text {bub }_{i}}\right)_{\Gamma^{e}}\right|
\end{aligned}
$$

where $l_{i}$ is the length of the edge $i$ in the element $\Omega^{e}$ and $(\tau)_{H^{1}}^{\boldsymbol{u}_{\mathcal{R}_{i}}}$ is the error time 
scale for each residual, i.e., for $i=M x, M y, C, S_{\perp}, S_{\|}$. In order to be consistent with the definition of the $\tau$ 's, we have included in each residual geometric factors of the measure of the element, $\left|\Omega^{e}\right|,[49,51,40]$. In Eq. (22) we can see that the residuals are projected into functions called $v_{\mathrm{bub}_{i}}$. The functions $v_{\mathrm{bub}_{i}}$ are $n_{\text {bub }}$ local bubble functions defined in the element and $n_{\text {edge }}$ on the element edges, which are related to the solution of the subgrid problem and error timescales, $\tau$ 's, see [55]. These functions are chosen to make sure that the local unit Stokes problems are solvable and stable, as the LBB condition requires. Finally, the strategy previously applied to Stokes is extended here to the Navier-Stokes equation. Note that the LBB is not affected by the convective term. These functions are defined as:

- Triangular elements: Let $\lambda_{T 1}, \lambda_{T 2}, \lambda_{T 3}$ be the barycentric or area coordinates. Then,

$$
\left\{\begin{array}{l}
v_{\mathrm{bub}_{i}}=\lambda_{T i} \cdot \lambda_{T j} \text { for } 1 \leq i<j \leq 3 \\
v_{\mathrm{bub}_{4}}=\lambda_{T 1} \lambda_{T 2} \lambda_{T 3}
\end{array}\right.
$$

Thus, we have four bubble functions per element.

- Quadrilateral elements: Let $\lambda_{Q 1}, \lambda_{Q 2}, \lambda_{Q 3}, \lambda_{Q 4}$ be the barycentric or area coordinates. Then,

$$
\left\{\begin{aligned}
v_{\mathrm{bub}_{i}} & =\frac{\lambda_{Q 1} \lambda_{Q 2} \lambda_{Q 3} \lambda_{Q 4}}{\lambda_{Q i}} \text { for } 1 \leq i \leq 4 \\
v_{\mathrm{bub}_{5}} & =\lambda_{Q 1} \lambda_{Q 2} \lambda_{Q 3} \lambda_{Q 4}
\end{aligned}\right.
$$

Thus, we have five bubble functions per element.

The rest of the section is devoted to explain the calculation of the $\tau$ 's. In this work, the error scales are obtained as a combination of asymptotic scales. This strategy has been used frequently and successfully to define stabilization parameters and error time-scales (see [29] for an example on stabilized methods and $[40,41]$ for error time-scales, where this approximation is explained and its impact on accuracy shown). Parameters defined from asymptotic limits are easier to obtain and less CPU demanding than exact closed forms. Sometines 
they are the only possible attainable expressions. Furthermore, for the incompressible Navier-Stokes equations we could have computed the error time-scales for each Reynolds number and each element, but using asymptotic scales, we avoid computing at the element level a local problem, which amounts to large computational savings.

Thus, in order to compute the error time-scales for the momentum equations, we consider two different contributions: one related to the convective term and another one connected to the solution of a local Stokes problem. The diffusiondominated contribution is taken from the work on Stokes flow [55], $\left(\tau_{S t}\right)_{H^{1}}^{\boldsymbol{u}_{\mathcal{R}_{i}}}$. The advection-dominated contribution is taken from the one-dimensional analysis of the advection-diffusion equation in [40] for the $H_{1}$ seminorm. There, it is shown that the error inverse-velocity scale for the transport equation is

$$
\frac{1}{\left|\boldsymbol{u}_{h}\right|} \min \left(\sqrt{\alpha}, \frac{\alpha}{\sqrt{3}}\right)
$$

where $\alpha=h_{e}\left|\boldsymbol{u}_{h}\right| /(2 \nu)$ is the element Reynolds number. Considering that the Stokes contribution already takes into account the diffusive limit, the final expression for the error scale can be simplified to

$$
(\tau)_{H^{1}}^{\boldsymbol{u}_{\mathcal{R}_{i}}}=\min \left(\frac{1}{\left|\boldsymbol{u}_{h}\right|} \frac{\alpha}{\sqrt{3}},\left(\tau_{S t}\right)_{H^{1}}^{\boldsymbol{u}_{\mathcal{R}_{i}}}\right)
$$

Once the elemental error is obtained, the global error (i.e. the error in the whole domain) can be computed as

$$
\left|\boldsymbol{u}^{\prime}\right|_{H^{1}(\Omega)}=\left(\sum_{\forall \Omega^{e} \in \Omega}\left|\boldsymbol{u}^{\prime}\right|_{H^{1}\left(\Omega^{e}\right)}^{2}\right)^{1 / 2}
$$

Remark 2. The Stokes error scales $\tau_{S t}$ 's represent a measure of the error on a element of unit area (measured in $H^{1}$-seminorm) produced by a determined unit residual. As quadrilaterals and triangles are employed, we have to compute ${ }_{225} \tau$ 's for both types of elements using a unit-area triangular domain and a unitarea rectangular domain, respectively. They are computed solving the problem below (28) on an element of unit area. That is, 
Find $\left(\boldsymbol{u}^{\prime}, p^{\prime}\right) \in\left(Q_{B}, P_{B}\right)$, that satisfies

$$
\left\{\begin{array}{cl}
\left(\nabla p^{\prime}, \boldsymbol{w}^{\prime}\right)+\nu\left(\nabla \boldsymbol{u}^{\prime}, \nabla \boldsymbol{w}^{\prime}\right)= & \\
(\boldsymbol{f}-\nabla \bar{p}+ & \left.\nu \nabla^{2} \overline{\boldsymbol{u}}, \boldsymbol{w}^{\prime}\right)_{\Omega^{e}}+\frac{1}{2}\left(\nu \llbracket \nabla \overline{\boldsymbol{u}} \cdot \boldsymbol{n} \rrbracket, \boldsymbol{w}^{\prime}\right)_{\Gamma^{e}}+ \\
(\boldsymbol{h}-\nu \nabla \overline{\boldsymbol{u}} & \left.\cdot \boldsymbol{n}, \boldsymbol{w}^{\prime}\right)_{\Gamma_{h}} \forall \boldsymbol{w}^{\prime} \in Q_{B} \\
\left(\nabla \cdot \boldsymbol{u}^{\prime}, q^{\prime}\right)= & \left(-\nabla \cdot \overline{\boldsymbol{u}}, q^{\prime}\right) \forall q^{\prime} \in P_{B}
\end{array}\right.
$$

where $Q_{B}$ and $P_{B}$ are the velocity and pressure spaces, respectively. The $\tau_{S t}$ 's are calculated integrating the solution of the subgrid problem. For more information, see $[55,76,56]$, where the shape functions are a combination of bubble functions and edge bubble functions. The selected finite element spaces for velocities, $Q_{B}$, and pressure, $P_{B}$, to solve the local problem (28), satisfy the Babuška-Brezzi condition.

Remark 3. The factor $\frac{1}{2}$ in the jump in Eq. (28), expresses the splitting of the residuals on the element boundary between the two elements that share the boundary $[10,3,56,76]$.

Remark 4. The values of the Stokes $\tau_{S t}$ 's are listed in Appendix A. Eq. (22) shows that a specific error scale corresponds to each type of residual. It turns out that the classic $\tau$ associated to the internal bubble is not enough to predict the error correctly. In fact, as can be seen in Appendix A, the $\tau_{S t}$ 's related to edge bubbles provide a significant error contribution (see [21], where it is shown that for low order elements, the edge residuals dominate the error estimate). Thus, a simpler error estimator could be considered taking into account only the residuals on the element boundaries, $\mathcal{R}_{S_{\perp}}$ and $\mathcal{R}_{S_{\|}}$.

245 Remark 5. The extension of the error estimation to 3D problems can be made using the expression (22) and considering the residuals on the faces instead of the residuals on the element boundaries. As a first approximation, the $\tau$ 's in Appendix A can be employed. 
Remark 6. Note that for estimates in the $H^{1}$ seminorm, the inviscid limit of the inverse-velocity error scale does not converge to the inviscid inversevelocity error scale. This is so because the slope of the solution in the layers increases as the viscosity decreases. As a consequence, the error scales in the $H^{1}$ seminorm cannot be composed from the error scales for the inviscid limit and those for the diffusive limit, but it has to be derived considering both

255 components simultaneously.

Remark 7. For incompressible and Stokes flow [55], direct computation of the error scales from the stabilization matrix underestimates the actual error. Also, we had to recur to numerical computation of the error constants because they could not be obtained from the fine-scale Green's function.

260 Remark 8. The error estimator presented here applies equally to the integratedby-parts form of the pressure gradient term of the momentum equations. In this case, the definition of the natural boundary condition has to be adapted.

\section{Adaptive mesh refinement}

Once the error is estimated we can identify the regions of the domain where the error is larger. Thus, the mesh can be modified in order to optimize the computational resources and concentrate the elements where it is necessary. The error estimate is measured in the $H^{1}$-seminorm as has been explained in Section 3. According to convergence theory, the norm of the error converges as $[48,29]$

$$
\left\|u^{\prime}\right\|_{H^{1}\left(\Omega^{e}\right)} \approx C h_{e}^{\beta}
$$

where $h_{e}$ is the mesh size, defined as the diameter of the circle having the same area as the area of the element. The coefficient $\beta$ is the convergence rate. Provided that the solution presents sufficient regularity and linear elements are employed, we have that $\beta=1$.

In order to create the new mesh with a new element size, the user establishes an error tolerance and the mesh must be modified in order to be close to the 
proposed tolerance. Thus, the relation between the new and old mesh size and the old and new error is as follows [37]

$$
\frac{h_{O L D}^{e}}{h_{N E W}^{e}}=\frac{\left\|u^{\prime}\right\|_{H^{1}\left(\Omega^{e}\right)}}{\left\|u_{T O L}^{\prime}\right\|_{H^{1}\left(\Omega^{e}\right)}}
$$

Following relation (30) and prescribing the error tolerance $\left\|u_{T O L}^{\prime}\right\|_{H^{1}\left(\Omega^{e}\right)}$, the desired element sizes are assigned to the old mesh, which becomes the background mesh. The refinement process has been performed by the GiD software [32], which generates an adapted mesh taking into consideration the newly provided element sizes and other inputs such as the geometry of the domain and the presence of distorted elements. In order to avoid overloading the mesh generator, a minimum mesh size of 0.005 is prescribed. As can be supposed, this is an iterative process in which we iterate until a satisfactory mesh is obtained. As depicted in Fig. 1, we summarize the adaptive mesh refinement in the following steps:

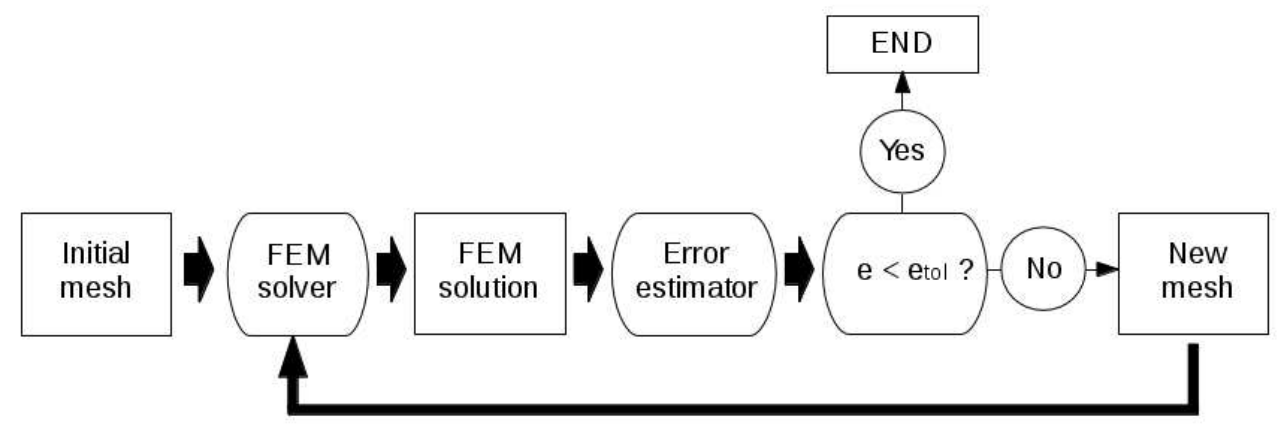

Figure 1: Flowchart for the adaptive mesh refinement. 
1 Taking a mesh, compute the FEM solution $\left(u_{h}, p_{h}, v_{h}\right)$ solving (10).

2 Estimate the elemental error $\left|\boldsymbol{u}^{\prime}\right|_{H^{1}\left(\Omega^{e}\right)}$ via the explicit method.

3 In case that the elemental error estimate, $\left\|u^{\prime}\right\|_{H^{1}\left(\Omega^{e}\right)}$, in each element is less than $\left\|u_{T O L}^{\prime}\right\|_{H^{1}\left(\Omega^{e}\right)}$, the process is stopped. Otherwise, go to the next step.

4 Taking into account the tolerance error, $\left\|u_{T O L}^{\prime}\right\|_{H^{1}\left(\Omega^{e}\right)}$, introduced by the user, we compute the new element size, $h_{N E W}^{e}$ by means of Eq. (30).

5 A new mesh is generated by GiD considering the new element sizes through a background mesh. Go back to step 1.

This procedure for adaptive mesh refinement is similar to the proposed by other authors, see for instance $[55,69,60,11,75]$. In the literature, other methodologies are available to generate adapted meshes. For example, starting from a initial mesh, other authors split the elements with large errors and merge those with small errors, $[66,34,22]$. In this latter case, usually there appear hanging nodes that must be treated. \\ 5. Numerical examples}

In this section three numerical problems are studied. The first numerical example is taken from [17], where a vortex rotates inside a square domain. This numerical example possesses an analytical solution, which depends on two constants that determine the position of a vortex in the square domain. The following two numerical examples are the lid-driven cavity and the backwardfacing step, which are classical benchmarks for the Navier-Stokes equations.

The numerical solution in the examples is obtained using the stabilized FEM formulation (10). Then, taking the FEM solution $\left(\boldsymbol{u}_{h}, p_{h}\right)$, the error is estimated. The quality of the error estimation is assessed by means of the concept of the efficiency index (see [3], for instance). It is the relation between the 
estimated error and the exact error,

$$
\text { eff }=\frac{\| \text { Estimated error } \|}{\| \text { Exact error } \|}
$$

where, in the absence of an analytical solution, the exact error is calculated using a reference solution computed on a fine mesh.

\subsection{Numerical test 1}

This numerical example is taken from Berrone [17], where a vortex is simulated in the square domain $(0,1) \times(0,1)$. The exact solution, which depends on the constants $R_{1}$ and $R_{2}$, can be written as

$$
\left\{\begin{array}{l}
u=\left(1-\cos \left(\frac{2 \pi\left(e^{R_{1} x}-1\right)}{e^{R_{1}}-1}\right)\right) \sin \left(\frac{2 \pi\left(e^{R_{2} y}-1\right)}{\left(e^{R_{2}}-1\right)}\right) \frac{R_{2}}{2 \pi} \frac{e^{R_{2} y}}{\left(e^{R_{2}}-1\right)} \\
v=-\sin \left(\frac{2 \pi\left(e^{R_{1} x}-1\right)}{\left(e^{R_{1}}-1\right)}\right)\left(1-\cos \left(\frac{2 \pi\left(e^{R_{2} y}-1\right)}{e^{R_{2}}-1}\right)\right) \frac{R_{1}}{2 \pi} \frac{e^{R_{1} x}}{\left(e^{R_{1}}-1\right)} \\
p=R_{1} R_{2} \sin \left(\frac{2 \pi\left(e^{R_{1} x}-1\right)}{\left(e^{R_{1}}-1\right)}\right) \sin \left(\frac{2 \pi\left(e^{R_{2} y}-1\right)}{\left(e^{R_{2}}-1\right)}\right) \frac{e^{R_{1} x} e^{R_{2} y}}{\left(e^{R_{1}}-1\right)\left(e^{R_{2}}-1\right)}
\end{array}\right.
$$

The load term $f$ is selected such that the above solution is recovered. This solution represents a counter-clockwise vortex, whose position depends on the parameters $R_{1}$ and $R_{2}$. The centre of the vortex has the coordinates $x_{0}=$ $1 / R_{1} \log \left(\left(e^{R_{1}}+1\right) / 2\right)$ and $y_{0}=1 / R_{2} \log \left(\left(e^{R_{2}}+1\right) / 2\right)$.

Three test cases are considered according to the following values of $R_{1}$ and $R_{2}$ :

- Test case 1: $R_{1}=R_{2}=0.1$

- Test case 2: $R_{1}=R_{2}=4$

- Test case 3: $R_{1}=4.2985$ and $R_{2}=0.1$

The solutions for these values of $R_{1}$ and $R_{2}$ are represented in Figs. 2-4. As has been commented the center of the vortex is modified with $R_{1}$ and $R_{2}$ and, also, the Reynolds number varies depending upon these constants [16]. 
Fig. 5 shows the convergence of exact error as a function of mesh element length for both, quadrilaterals and triangles. It is observed that the convergence rate is 1 for the velocity error measured in the $H^{1}$-seminorm. In subfigures b) and c) we can see that the error is not reduced properly until the element size is small enough to capture the vortex.

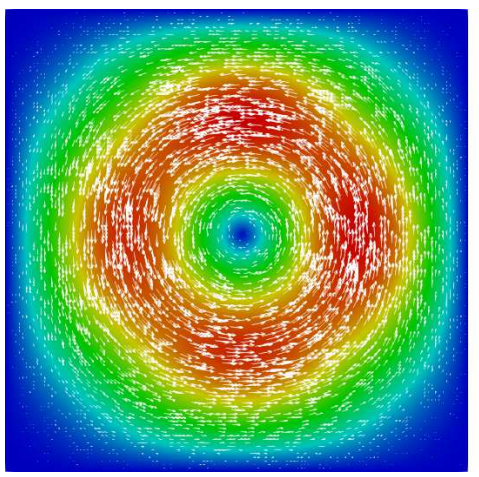

(a) Velocity field

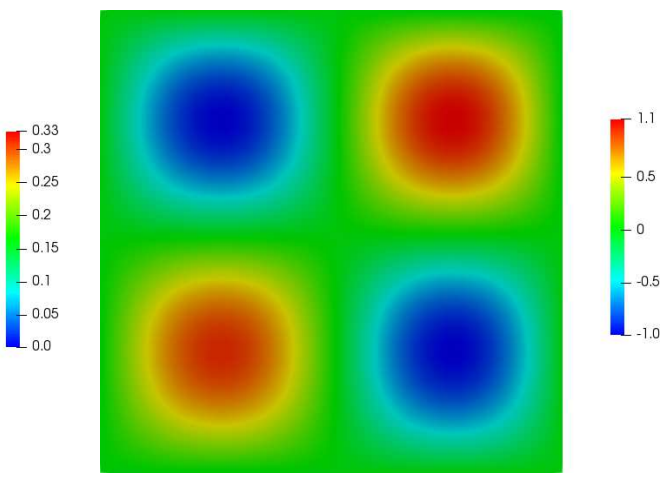

(b) Pressure

Figure 2: Numerical test 1 . Velocity field and pressure. Test case $1: R_{1}=0.1, R_{2}=0.1$.

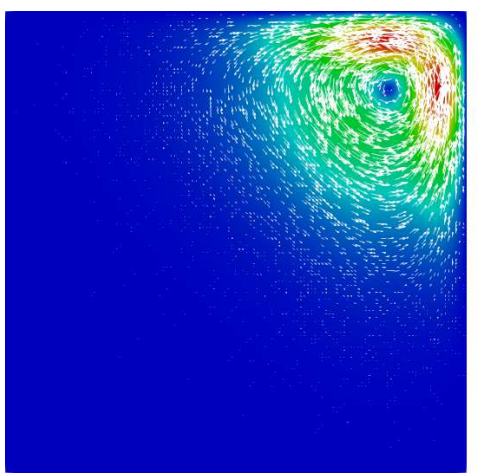

(a) Velocity field

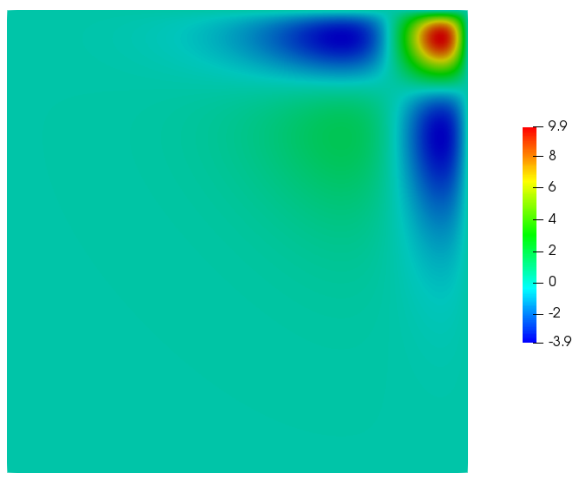

(b) Pressure

Figure 3: Numerical test 1. Velocity field and pressure. Test case 2: $R_{1}=4, R_{2}=4$.

In order to assess the behavior of our estimator with element length, uniform meshes with various number of elements are employed. Global efficiencies are shown in Table 1 for the three test cases and various meshes.

As for the $H^{1}$ seminorm of the velocity error, the local estimated and exact 


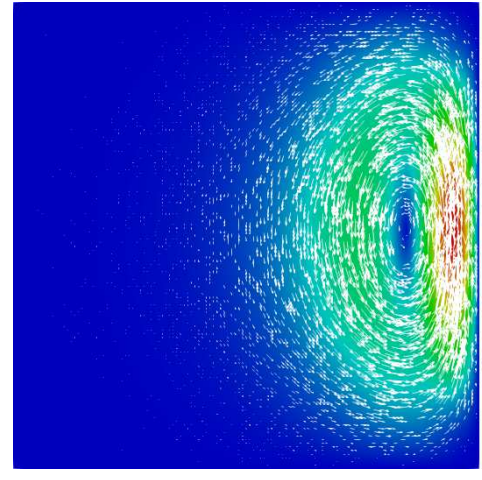

(a) Velocity field

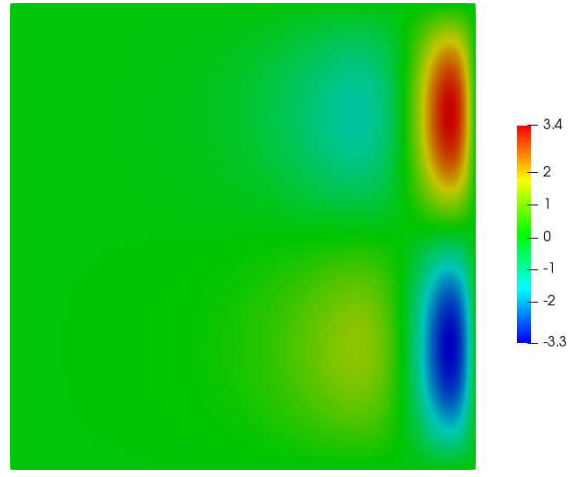

(b) Pressure

Figure 4: Numerical test 1 . Velocity field and pressure. Test case $3: R_{1}=4.2985, R_{2}=0.1$

\begin{tabular}{lccc|lccc}
\hline & \multicolumn{4}{c}{ Quadrilaterals } & \multicolumn{4}{c}{ Triangles } \\
$n_{e l}$ & Test 1 & Test 2 & Test 3 & $n_{e l}$ & Test 1 & Test 2 & Test 3 \\
\hline 16 & 4.363 & 0.950 & 1.201 & 16 & 1.252 & 0.288 & 0.464 \\
64 & 6.766 & 1.508 & 1.588 & 64 & 1.687 & 0.495 & 0.496 \\
256 & 7.557 & 2.974 & 2.088 & 256 & 1.917 & 0.959 & 0.753 \\
1024 & 7.801 & 4.359 & 3.267 & 1024 & 2.022 & 1.207 & 0.870 \\
4096 & 7.883 & 5.073 & 3.840 & 1024 & 2.071 & 1.347 & 0.947 \\
\hline
\end{tabular}

Table 1: Numerical test 1. Global efficiencies for velocity in $H^{1}$-seminorm for quadrilaterals and triangles.

error norms are shown for the three test cases in Figs. 6-8. 


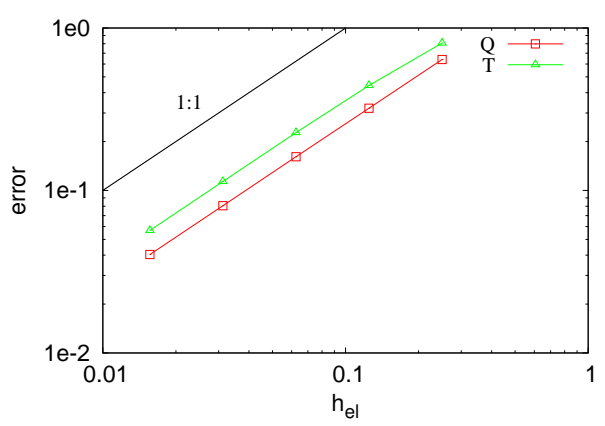

(a) Test 1

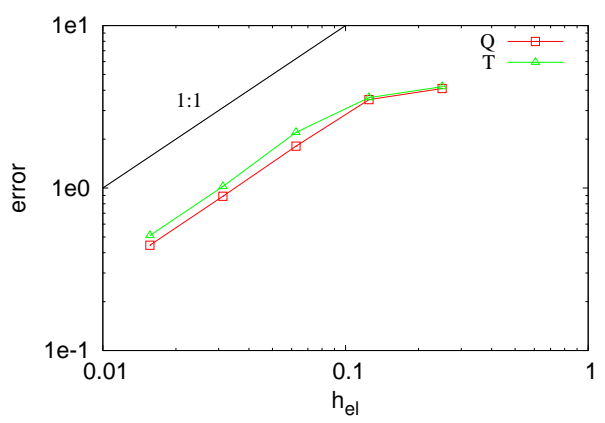

(b) Test2

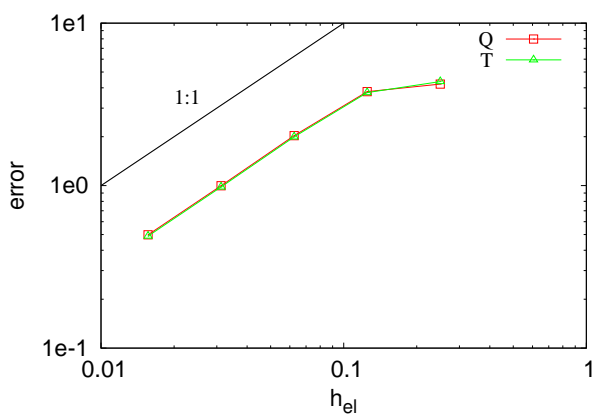

(c) Test 3

Figure 5: Numerical test 1. Error convergence $\left|\boldsymbol{u}^{\prime}\right|_{H^{1}}$ for the three proposed cases using quadrilaterals $(\mathrm{Q})$ and triangles $(\mathrm{T})$. 


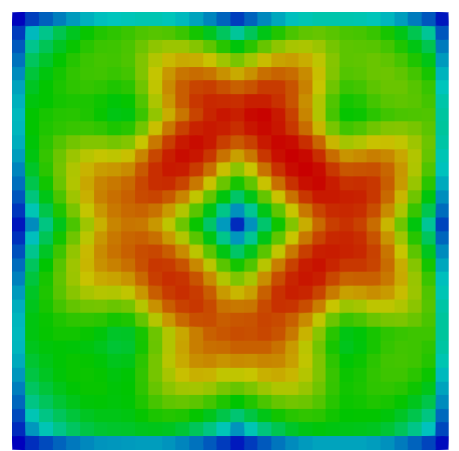

(a) Estimated error

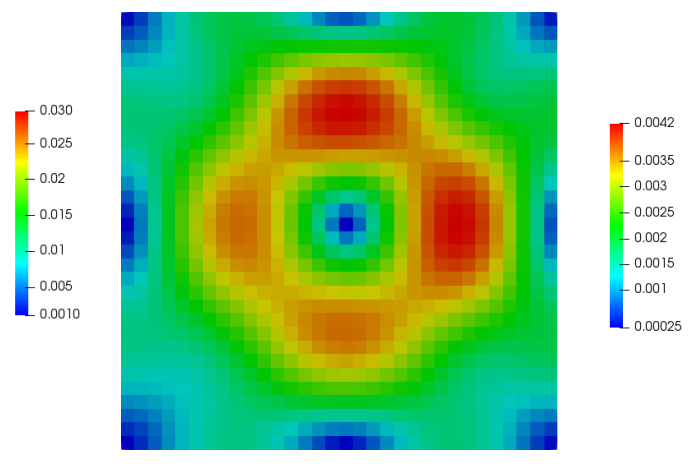

(b) Exact error

Figure 6: Numerical test 1. Estimated and exact error. Test case 1: $R_{1}=0.1, R_{2}=0.1$

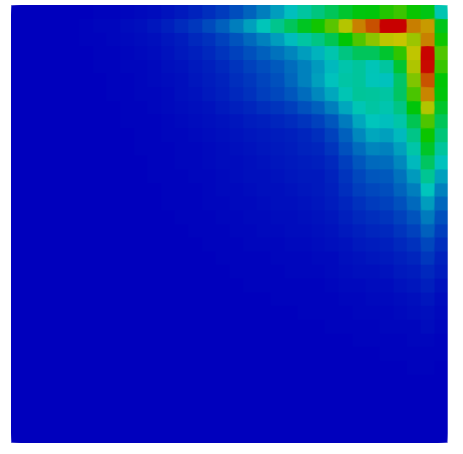

(a) Estimated error

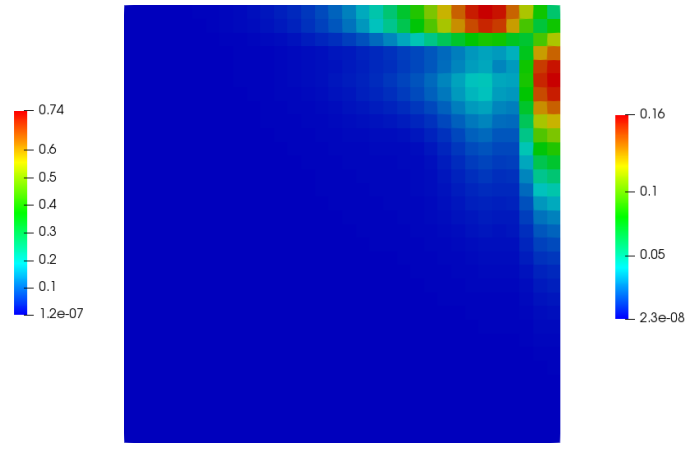

(b) Exact error

Figure 7: Numerical test 1. Estimated and exact error. Test case 2: $R_{1}=4, R_{2}=4$.

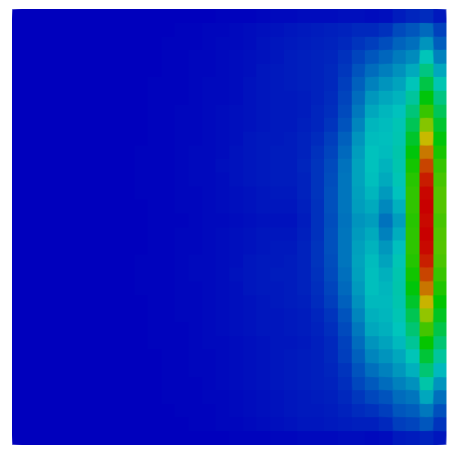

(a) Estimated error

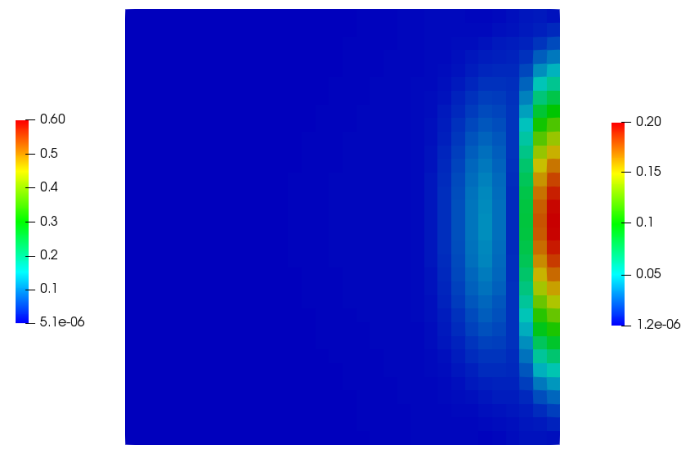

(b) Exact error

Figure 8: Numerical test 1 . Estimated and exact error. Test case $3: R_{1}=4.2985, R_{2}=0.1$. 
Using the local error estimates, the mesh refinement process described beforehand in Section 4 is followed. The point of departure is a uniform mesh and then, the refinement iterative process is applied. The generated adapted meshes for Test cases 2 and 3 can be seen in Figs. 9 to 12 for an error tolerance of $\left\|u_{T O L}^{\prime}\right\|_{H^{1}\left(\Omega^{e}\right)}=0.05$. From them, it can be seen that element sizes evolve very smoothly and become smaller in regions where the solution is more abrupt.

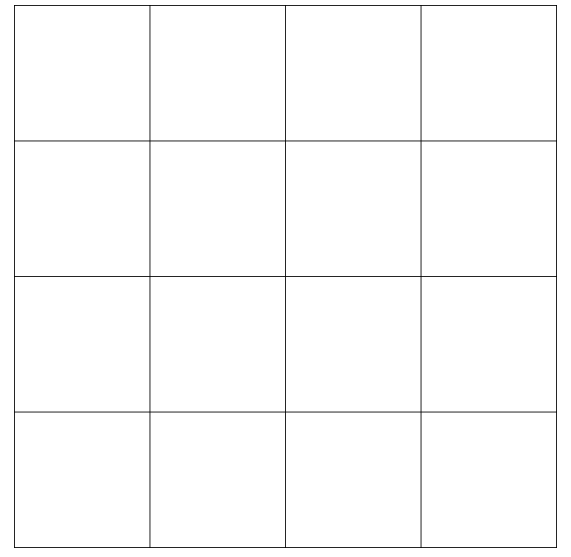

(a) Initial mesh

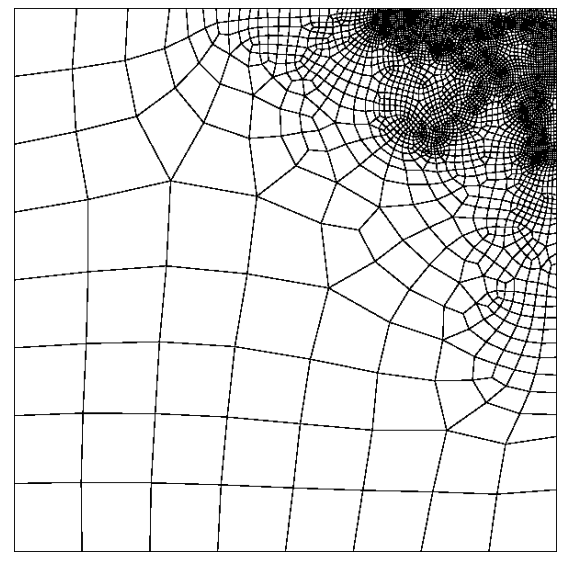

(b) Final mesh

Figure 9: Numerical test 1 . Adaptive mesh refinement for quadrilaterals. Test case 2: $R_{1}=$ $4.0, R_{1}=4.0$.

We can observe that, for $R_{1}=4.2985$ and $R_{2}=0.1$, in the final mesh the elements are concentrated where the error is greater, that is, on the right edge of the domain. However, for $R_{1}=4.0$ and $R_{2}=4.0$, the elements are concentrated at the top right corner, where there exist large gradients of the solution.

In order to show the improvement of the solution using mesh refinement, Tables 2 and 3 represent the global error and the maximum local error for a uniform mesh and a refined mesh with a similar number of elements. It can be seen that the error is reduced both globally and locally using the refined mesh.

Furthermore, Fig. 13 shows log-log plots of the convergence with respect to element length of the absolute exact and estimated global errors for the three test cases with quads and triangles. Note that in all cases, the $H^{1}$ seminorm of 


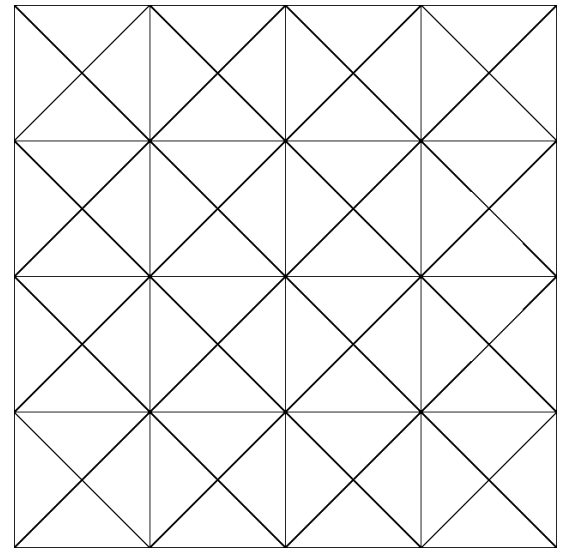

(a) Initial mesh

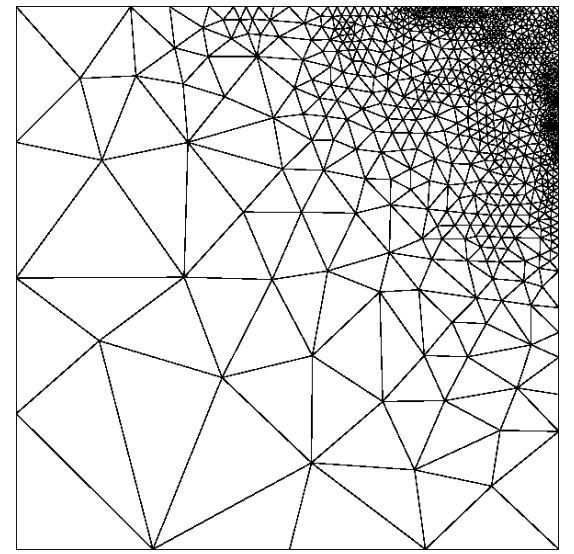

(b) Final mesh

Figure 10: Numerical test 1. Adaptive mesh refinement for triangles. Test case 2: $R_{1}=4.0$, $R_{1}=4.0$.

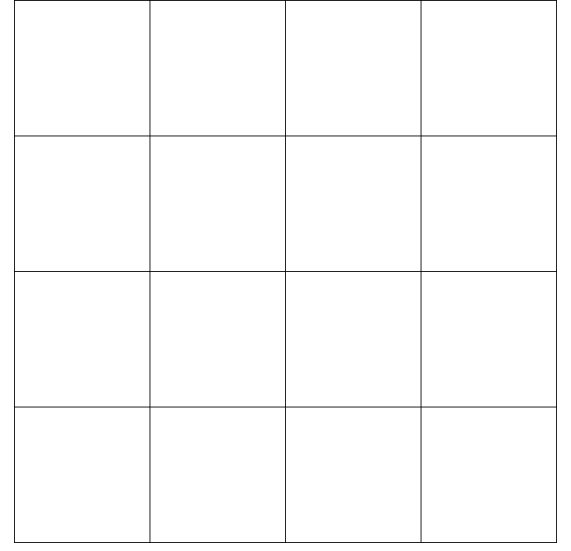

(a) Initial mesh

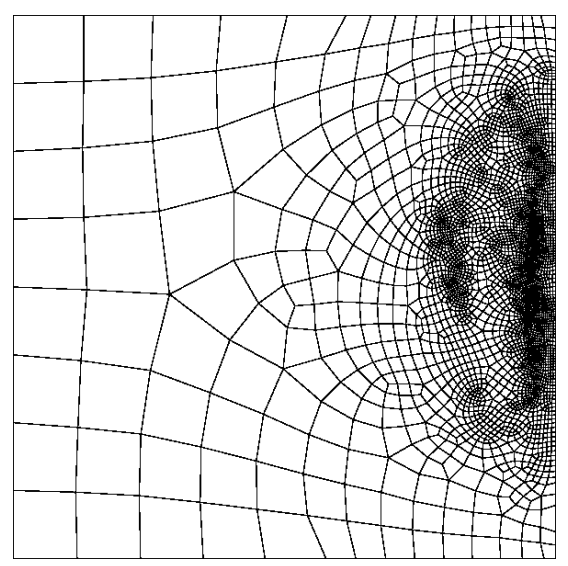

(b) Final mesh

Figure 11: Numerical test 1. Adaptive mesh refinement for quadrilaterals. Test case 3: $R_{1}=4.2985, R_{1}=0.1$.

the velocity errors converges at the expected rate of 1 . More importantly, the estimated error norm converges at the theoretical rate of 1 , proving that the method is an error estimator (and not a simpler error indicator).

In the present method, the steady state finite element solution accuracy 


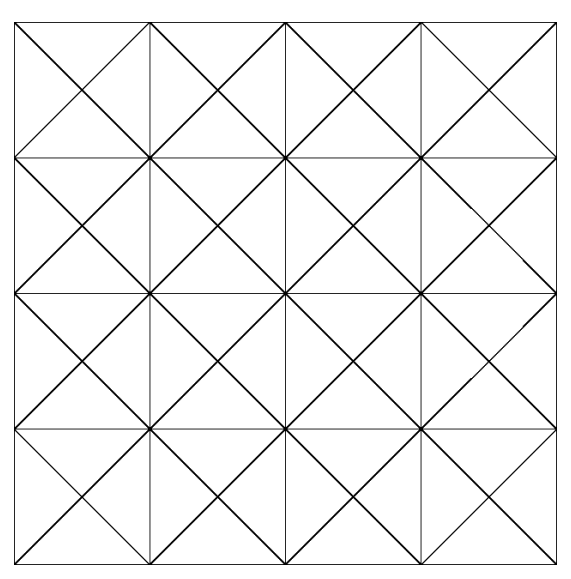

(a) Initial mesh

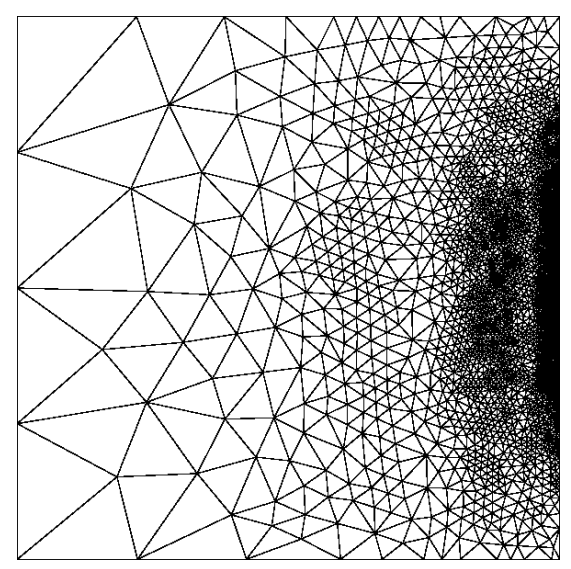

(b) Final mesh

Figure 12: Numerical test 1. Adaptive mesh refinement for triangles. Test case $3: R_{1}=$ 4.2985, $R_{2}=0.1$.

\begin{tabular}{|c|c|c|c|c|c|c|c|}
\hline \multicolumn{4}{|c|}{ Quadrilaterals } & \multicolumn{4}{|c|}{ Triangles } \\
\hline Mesh & $n_{e l}$ & $\begin{array}{l}\text { Global } \\
\text { error }\end{array}$ & $\begin{array}{l}\text { maximum } \\
\text { local error }\end{array}$ & Mesh & $n_{e l}$ & $\begin{array}{c}\text { Global } \\
\text { error }\end{array}$ & $\begin{array}{l}\text { maximum } \\
\text { local error }\end{array}$ \\
\hline Uniform & 3884 & $4.65 \mathrm{E}-1$ & $4.53 \mathrm{E}-2$ & Uniform & 8192 & $5.11 \mathrm{E}-1$ & $3,67 \mathrm{E}-2$ \\
\hline Refined & 3778 & $1.78 \mathrm{E}-1$ & $1.22 \mathrm{E}-2$ & Refined & 7805 & $1.59 \mathrm{E}-1$ & $7,35 \mathrm{E}-3$ \\
\hline
\end{tabular}

Table 2: Numerical test 1. Comparison of global and local error for the uniform and refined meshes. $R_{1}=4, R_{2}=4$

\begin{tabular}{lccc|lcccc}
\hline \multicolumn{3}{c}{ Quadrilaterals } \\
Mesh & \multicolumn{3}{c}{$\begin{array}{c}\text { Global } \\
n_{e l}\end{array}$} & $\begin{array}{c}\text { maximum } \\
\text { error }\end{array}$ & local error & Mesh & & \multicolumn{3}{c}{ Triangles } \\
$n_{e l}$ & $\begin{array}{c}\text { Global } \\
\text { error }\end{array}$ & $\begin{array}{c}\text { maximum } \\
\text { local error }\end{array}$ \\
\hline Uniform & 4096 & $4.98 \mathrm{E}-1$ & $5.29 \mathrm{E}-2$ & Uniform & 8192 & $4.89 \mathrm{E}-1$ & $3,76 \mathrm{E}-2$ \\
Refined & 4107 & $1.36 \mathrm{E}-1$ & $9.45 \mathrm{E}-3$ & Refined & 7890 & $1.15 \mathrm{E}-1$ & $8.01 \mathrm{E}-3$ \\
\hline
\end{tabular}

Table 3: Numerical test 1. Comparison of global and local error for the uniform and refined meshes. $R_{1}=4.2985, R_{2}=0.1$

depends on the number of passes of the predictor-multicorrector algorithm and the tolerance of the iterative solver. Since we are searching for the steady state, the number of passes has been set to 1 . So this leaves the tolerance of the 


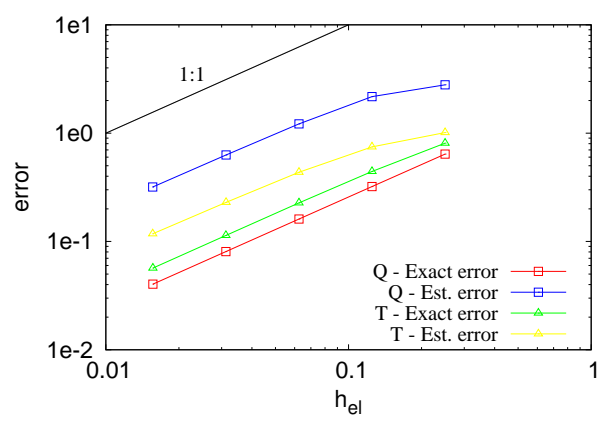

(a) Test case 1

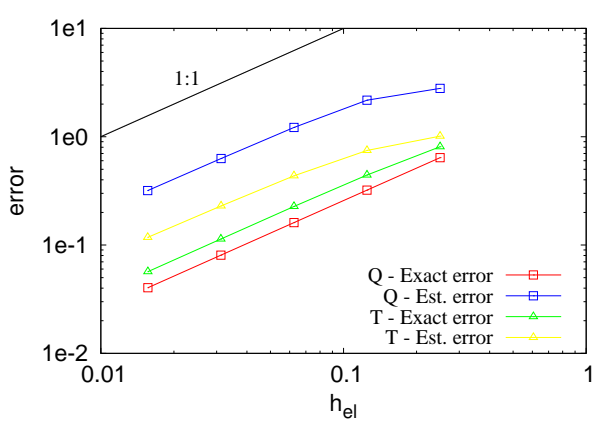

(b) Test case 2

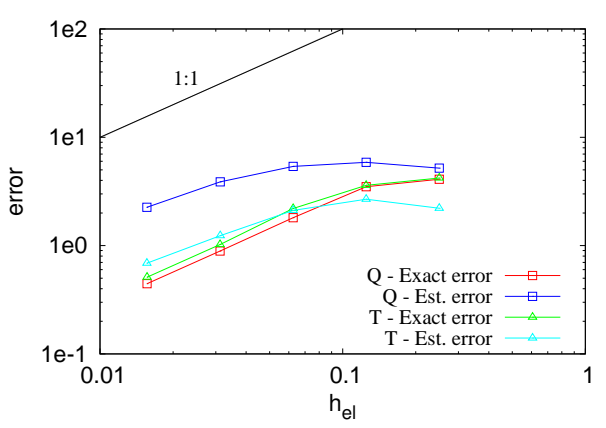

(c) Test case 3

Figure 13: Numerical test 1. Convergence of exact and estimated global error $\left|\boldsymbol{u}^{\prime}\right|_{H^{1}}$ as a function of element length

iterative solver as the remaining parameter relevant to the solution accuracy for a large number of time steps. Thus, for test case 1, Fig. 14 shows the 


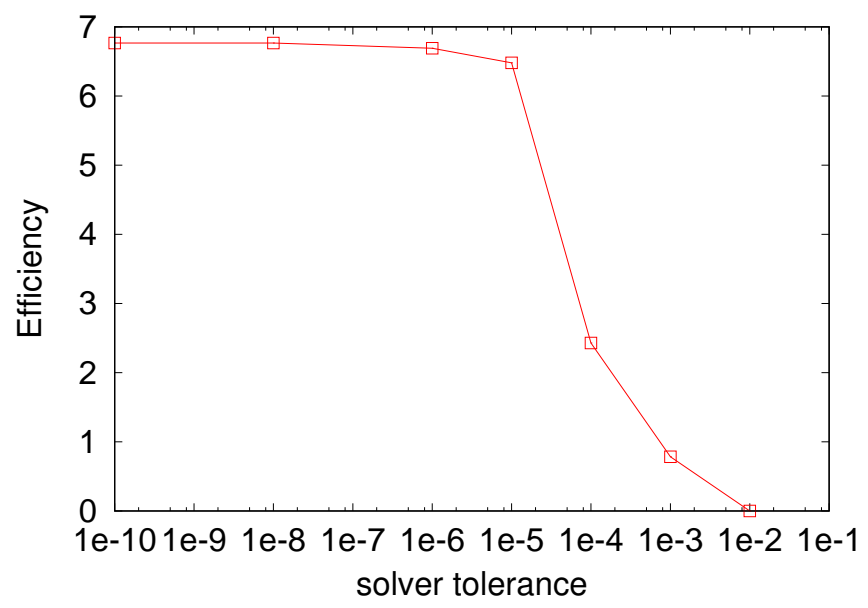

Figure 14: Numerical test 1, case 1. Influence of the iterative solver tolerance on efficiency.

influence of the iterative solver error tolerance on efficiency. It shows that for error tolerances smaller than $1 \cdot 10^{-5}$, which is a reasonable value, the efficiency becomes independent of this parameter. In general, the methods assumes that the numerical solution satisfies the discrete equations, as happens with many 350 other error estimators. 


\subsection{Numerical test 2: Lid-driven cavity}

The lid-driven cavity problem is a typical benchmark for viscous fluid flows. The domain is a unitary square consisting of three edges with no-slip conditions and a top edge with a unit tangential velocity (see Fig. 15). The pressure is set to zero at the lower left corner. As usual, the Reynolds number is based on the lid velocity and the square side length. Uniform meshes are considered.

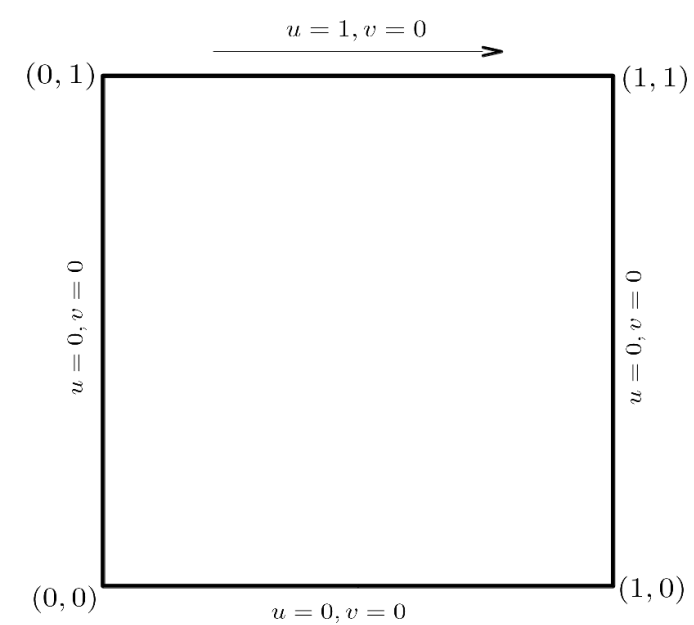

Figure 15: Lid-driven cavity flow problem with unitary velocity on the top edge.

\begin{tabular}{lccc|lccc}
\hline & \multicolumn{3}{c}{ Quadrilaterals } & \multicolumn{3}{c}{ Triangles } \\
$n_{e l}$ & $\mathrm{Re}=1$ & $\mathrm{Re}=100$ & $\mathrm{Re}=1000$ & $n_{e l}$ & $\mathrm{Re}=1$ & $\mathrm{Re}=100$ & $\mathrm{Re}=1000$ \\
\hline 16 & 2.717 & 2.482 & 2.433 & 32 & 0.845 & 0.825 & 0.795 \\
64 & 3.404 & 2.798 & 2.048 & 128 & 1.192 & 1.136 & 1.125 \\
256 & 3.305 & 2.693 & 1.887 & 512 & 1.263 & 1.150 & 0.932 \\
1024 & 3.843 & 3.294 & 2.166 & 2048 & 1.294 & 1.210 & 0.921 \\
\hline
\end{tabular}

Table 4: Numerical test 2. Global efficiencies for velocity in $H^{1}$-seminorm for quadrilaterals and triangles.

Table 4 shows the global efficiencies for the considered estimators whereas Fig. 16 represents the local efficiencies for $n_{e l}=16$ and $n_{e l}=256$.

Once the local error is estimated, we can evaluate where it is convenient to 


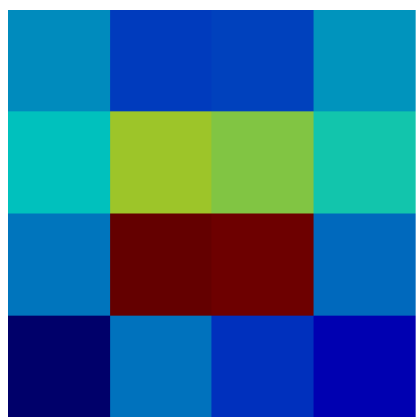

(a) $n_{e l}=16$

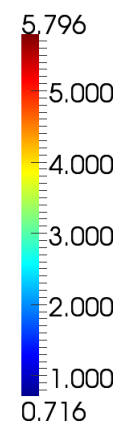

1.00

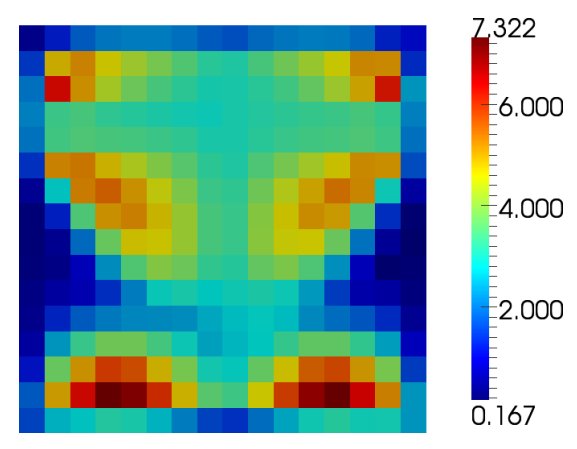

Figure 16: Numerical test 2. Local efficiencies with the explicit error estimator. $R e=1$

refine the mesh to obtain a more accurate solution. This remeshing process is made following Section 4 , with an objective error tolerance of $\left\|u_{T O L}^{\prime}\right\|_{H^{1}\left(\Omega^{e}\right)}=$ 0.080. In Figs. 17 and 18, we observe how the elements are concentrated at the upper corners. The greater the Reynolds number, the finer the elements on the right side.

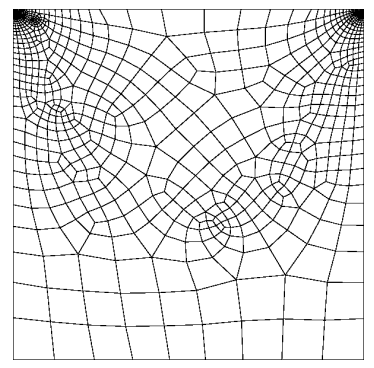

(a) $\mathrm{Re}=1$

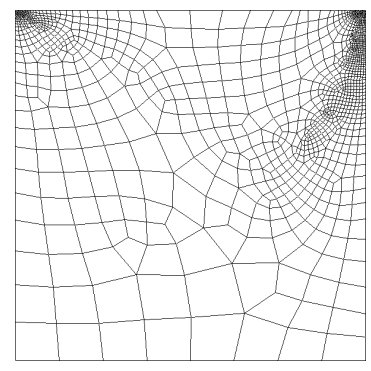

(b) $\mathrm{Re}=100$

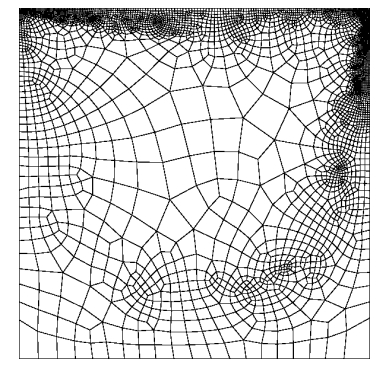

(c) $\operatorname{Re}=1000$

Figure 17: Numerical test 2. Adaptive mesh refinement for quadrilaterals. $R e=1, R e=100$ and $\operatorname{Re}=1000$

\subsection{Numerical test 3: Backward-Facing Step}

This is another classical benchmark for the Navier-Stokes equations (see Fig. 19 for the problem setup). We examine the error estimator for the Reynolds numbers $\operatorname{Re}=1, \operatorname{Re}=10$ and $R e=100$. Homogeneous Dirichlet boundary conditions are set on the boundary except on the edge $\overline{A B}$ where parabolic inflow 


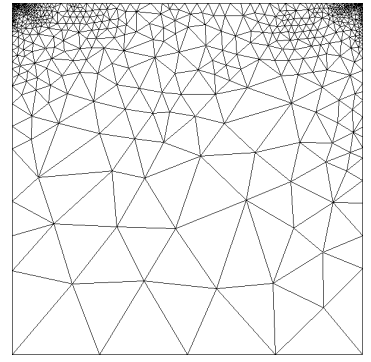

(a) $\mathrm{Re}=1$

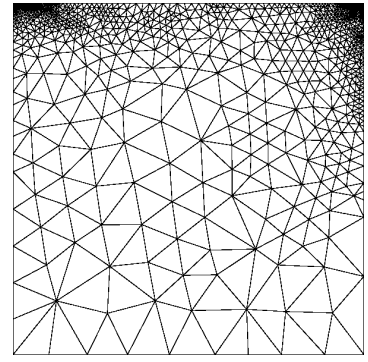

(b) $\operatorname{Re}=100$

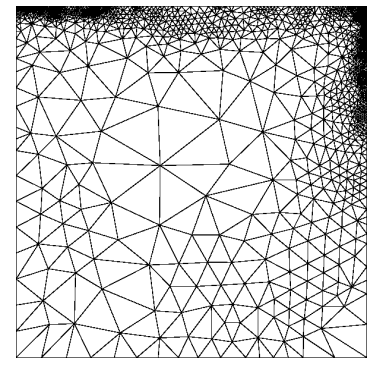

(c) $\mathrm{Re}=1000$

Figure 18: Numerical test 2. Adaptive mesh refinement for triangles. $R e=1, R e=100$ and $\operatorname{Re}=1000$

boundary conditions are considered and on the edge $\overline{C D}$ with natural outflow boundary conditions. All the quasi-uniform meshes have been generated with GiD [32].

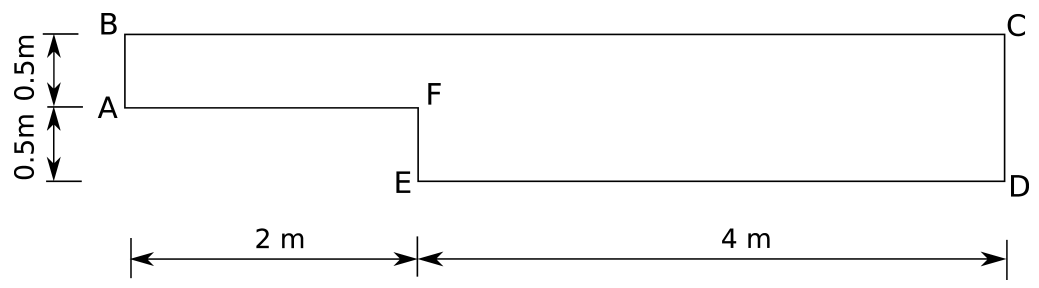

Figure 19: Numerical test 3. Backward facing step problem setup.

\begin{tabular}{lccc|cccc}
\hline \multicolumn{4}{c}{ Quadrilaterals } & \multicolumn{3}{c}{ Triangles } \\
$n_{e l}$ & $R e=1$ & $R e=10$ & $R e=100$ & $n_{e l}$ & $R e=1$ & $R e=10$ & $R e=100$ \\
\hline 320 & 2.609 & 2.598 & 2.628 & 640 & 1.301 & 1.312 & 1.755 \\
1280 & 3.626 & 3.575 & 3.403 & 1280 & 1.449 & 1.342 & 1.138 \\
2343 & 3.928 & 3.841 & 3.554 & 2560 & 1.528 & 1.604 & 1.345 \\
5120 & 4.561 & 4.499 & 4.274 & 5120 & 1.987 & 1.962 & 1.460 \\
\hline
\end{tabular}

Table 5: Numerical test 3. Global efficiencies for velocity in $H^{1}$-seminorm for quadrilaterals and triangles.

Following Biswas [18], the Reynolds number is defined as $R e=\frac{U_{b} 2 H}{\nu}$, where $U_{b}$ is mean velocity in the inlet, $H$ is the inlet width and $\nu$ is the kinematic 


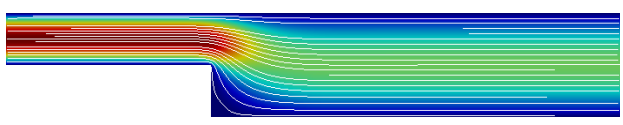

(a) $R e=1$

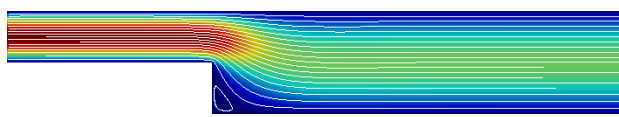

(b) $R e=10$

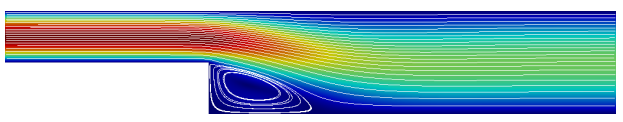

(c) $R e=100$

Figure 20: Numerical test 3. Velocity magnitude and streamlines over the step for $\mathrm{Re}=1$, $\operatorname{Re}=10$ and $\operatorname{Re}=100$.

viscosity.

The numerical solution is represented in Fig. 20 for the three Reynolds numbers. Local $H^{1}$ seminorm error estimates and efficiencies are represented in Figs. 21 and 22 for $R e=1$ and $R e=100$ on the mesh of 1280 elements. As expected, the error is greater at the inner corner in both cases. The efficiencies are satisfactory in the whole domain. The error estimator predicts correctly the regions where the error is larger. Global efficiencies are shown in Table 5 for various meshes and Reynolds numbers.

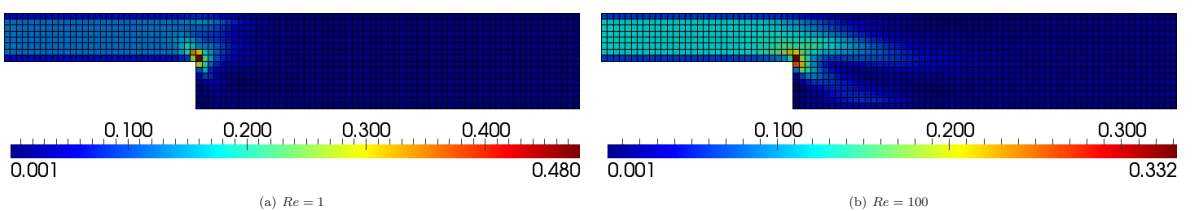

Figure 21: Numerical test 3. Local error estimates for $\mathrm{Re}=1$ and $\mathrm{Re}=100$.
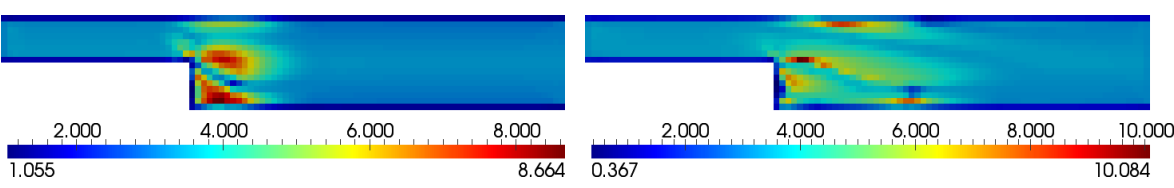

(a) $R e=1$

(b) $R e=100$

Figure 22: Numerical test 3. Local efficiencies for $\operatorname{Re}=1$ and $\operatorname{Re}=100$. 
Fig. 23 shows the initial mesh and the adapted meshes for Reynolds numbers 1 and 100 generated with the algorithm of Section 4 for an error tolerance of $385\left\|u_{T O L}^{\prime}\right\|_{H^{1}\left(\Omega^{e}\right)}=0.025$. Note that the elements are concentrated around the step corner.

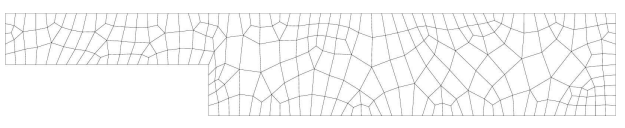

(a) Initial mesh

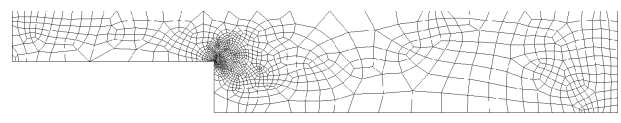

(b) Final mesh. $\mathrm{Re}=1$

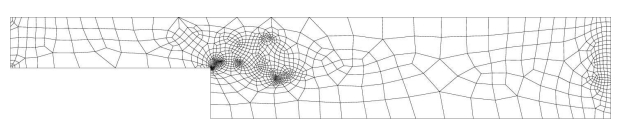

(c) Final mesh. Re $=100$

Figure 23: Numerical test 3. Mesh adaptivity for $R e=1$ and $R e=100$. 


\section{Conclusion}

An explicit a posteriori error estimator initially developed for Stokes flow has been extended to the incompressible Navier-Stokes equations. The method 390 is based on the combination of error time (inverse-velocity) scales and residuals, including element interior residuals and egde residuals. The error time scales are a-priori computed parameters, very much like the stabilization parameters, but containing error information. Considering that the technology is explicit, it has been seen to give reasonable values for the global and local efficiencies and that

395 the estimated error converges at the theoretical convergence rate. Furthermore, it has been shown that, compared to uniform refiment, the error decreases faster with adapted mesh refinement. Also, the refinement process can be seen to provide smooth adapted meshes with a control of the target local error norm. Finally, the method is robust with respect to the tolerance of the iterative

400 solver (embedded within the nonlinear solver), since the error estimates become independent for tolerances smaller than $10^{-5}$.

\section{Acknowledgements}

This work has been partially funded by the Ministerio de Economía y Competitividad under contract PID2019-106099RB-C44 (AEI/FEDER,UE), Gob-

405 ierno de Aragón/FEDER-UE (Grupo de Investigacion de Referencia de Mecanica de Fluidos Computacional T32_20R). 


\section{Appendix A. Stokes error inverse-velocity scales}

The error inverse-velocity scales, $\tau$ 's, are parameters connected to the variational multiscale theory. Typically they represent an average of the fine scales and provide information about how the fine scales affect the coarse scales. Regarding error estimation, these error scales link the residuals to the error.

For the Stokes problem, we can distinguish between internal residuals $\left(\mathcal{R}_{M x}\right.$, $\mathcal{R}_{M y}$ and $\left.\mathcal{R}_{C}\right)$ and inter-element residuals $\left(\mathcal{R}_{S_{\perp}}\right.$ and $\left.\mathcal{R}_{S_{\|}}\right)$. To each type of residual, a specific error scale was derived in [55], which are collected in Table A.6 for quadrilaterals and triangles.

\begin{tabular}{l|ccccc|} 
Element & $\left(\tau_{S t}\right)_{H^{1}}^{\boldsymbol{u}_{\mathcal{R}_{M x}}}$ & $\left(\tau_{S t}\right)_{H^{1}}^{\boldsymbol{u}_{\mathcal{R}_{M y}}}$ & $\left(\tau_{S t}\right)_{H^{1}}^{\boldsymbol{u}_{\mathcal{R}_{C}}}$ & $\left(\tau_{S t}\right)_{H^{1}}^{\boldsymbol{u}_{\mathcal{R}_{\perp}}}$ & $(\tau)_{H^{1}}$ \\
\hline Quadrilaterals & $\frac{h_{e}}{\nu} 5.7735 \mathrm{e}-2$ & $\frac{h_{e}}{\nu} 5.7735 \mathrm{e}-2$ & $9.53462 \mathrm{e}-1$ & $\frac{1}{\nu} 2.41680 \mathrm{e}-1$ & $\frac{1}{\nu} 5.74456 \mathrm{e}-1$ \\
\hline Triangles & $\frac{h_{e}}{\nu} 2.41263 \mathrm{e}-1$ & $\frac{h_{e}}{\nu} 2.08712 \mathrm{e}-1$ & $2.79196 \mathrm{e}-0$ & $\frac{1}{\nu} 4.07206 \mathrm{e}-1$ & $\frac{1}{\nu} 2.53842 \mathrm{e}-1$ \\
\hline
\end{tabular}

Table A.6: Stokes error inverse-velocity scales employed in explicit error estimation for quadrilaterals and triangles

415

Remark 9. Following the philosophy of stabilized methods, the units of the $\tau$ 's are independent of the problem dimension. For 2D problems, the element size is $h_{e}=\sqrt{\Omega^{e}}$ and for $3 \mathrm{D} h_{e}=\sqrt[3]{\Omega^{e}}$.

[1] Ahmed, N., Chacon-Rebollo, T., John, V., Rubino, S.: A review of variational multiscale methods for the simulation of turbulent incompressible flows. Arch Computat Methods Eng 24, 115-164 (2017)

[2] Ainsworth, M., Oden, J.T.: A posterior error estimates for Stokes' and Oseen's equations. SIAM J. Numer. Anal. 34, 228-245 (1997)

[3] Ainsworth, M., Oden, J.T.: A posterior error estimation in finite element analysis. John Wiley \& Sons (2000)

[4] Araya, R., Barrenechea, G.R., Valentin, F.: Stabilized finite element methods based on multiscale enrichment for the Stokes problem. SIAM Journal on Numerical Analysis 44(1), 322-348 (2006) 
[5] Araya, R., Behrens, E., Rodriguez, R.: Error estimator advection-reactiondiffusion equations based on solution of local problems. Applied Numerical Mathematics 206, 440-453 (2007)

[6] Araya, R., Valentin, F.: A multiscale a posteriori error estimate. Comput. Meth. Appl. Mech. Engrg. 194, 2077-2094 (2005)

[7] Babuška, I.: The finite element method with Lagrangian multipliers. Numerische Mathematik 20(3), 179-192 (1973)

[8] Baiges, J., , Codina, R.: Variational multiscale error estimators for solid mechanics adaptive simulations: An orthogonal subgrid scale approach. Computer Methods in Applied Mechanics and Engineering 325, 37-55 (2017)

[9] Bangerth, W., Rannacher, R.: Adaptive finite element methods for differential equations. Birkhäuser (2003)

[10] Bank, R., Weiser, A.: Some a posteriori error estimators for elliptic partial differential equations. Math. Comp. 44, 283-301 (1985)

[11] Bank, R.E., Welfert, B.D.: A posteriori error estimates for the Stokes problem. SIAM Journal on Numerical Analysis 28(3), 591-623 (1991)

[12] Bayona-Roa, C., Codina, R., Baiges, J.: Variational multiscale error estimators for the adaptive mesh refinement of compressible flow simulations. Computer Methods in Applied Mechanics and Engineering 337, 501-526 (2018)

[13] Bazile, A., Hachem, E., Larroya-Huguet, J., Mesri, Y.: Variational multiscale error estimator for anisotropic adaptive fluid mechanic simulations: Application to convection-diffusion problems. Computer Methods in Applied Mechanics and Engineering 331, 94-115 (2018)

[14] Bazilevs, Y., Calo, V., Cottrell, J., Hughes, T., Reali, A., Scovazzi, G.: Variational multiscale residual-based turbulence modeling for large eddy 
simulation of incompressible flows. Computer Methods in Applied Mechanics and Engineering 197(1), 173 - 201 (2007)

[15] Behr, M., Tezduyar, T.: Finite element solution strategies for large-scale flow simulations. Computer Methods in Applied Mechanics and Engineering 112(1-4), 3-24 (1994)

[16] Berrone, E.: Robustness in a posteriori error analysis for FEM flow models. Numer. Math. 91, 389-422 (2002)

[17] Berrone, S.: Adaptive discretization of stationary and incompressible Navier-Stokes equations by stabilized finite element methods. Computer Methods in Applied Mechanics and Engineering 190(34), 4435-4455 (2001)

[18] Biswas, G., Breuer, M., Durst, F.: Backward-facing step flows for various expansion ratios at low and moderate reynolds numbers. Journal of fluids engineering 126(3), 362-374 (2004)

[19] Brezzi, F.: On the existence, uniqueness and approximation of saddlepoint problems arising from Lagrangian multipliers. Revue française d'automatique, informatique, recherche opérationnelle. Analyse numérique 8(2), 129-151 (1974)

[20] Brezzi, F., Pitkäranta, J.: On the stabilization of finite element approximations of the Stokes equations. Springer (1984)

475 [21] Carstensen, C., Verfürth, R.: Edge residuals dominate a posteriori error estimates for low order finite element methods. SIAM J. Numer. Anal. 36(5), 1571-1587 (1999)

[22] Cirak, F., Ramm, E.: A posteriori error estimation and adaptivity for linear elasticity using the reciprocal theorem. Computer Methods in Applied Mechanics and Engineering 156(1-4), 351-362 (1998)

[23] Codina, R.: Stabilization of incompressibility and convection through orthogonal sub-scales in finite element methods. Computer methods in applied mechanics and engineering 190(13-14), 1579-1599 (2000) 
[24] Codina, R., Badia, S., Baiges, J., Principe, J.: Variational multiscale methods in computational fluid dynamics. Encyclopedia of Computational Mechanics pp. 1-28 (2017)

[25] Colomes, O., Scovazzi, G., Guilleminot, J.: On the robustness of variational multiscale error estimators for the forward propagation of uncertainty. Comput. Methods Appl. Mech. Engrg. 342, 384-413 (2018)

[26] Colomes, O., Scovazzi, G., Sraj, I., Knio, O., Le Maître, O.: A finite volume error estimator inspired by the variational multiscale approach. In: 2018 AIAA Non-Deterministic Approaches Conference, p. 1178 (2018)

[27] Dettmer, W., Peric, D.: An analysis of the time integration algorithms for the finite element solutions of incompressible NavierStokes equations based on a stabilised formulation. Computer Methods in Applied Mechanics and Engineering 192(9), 1177 - 1226 (2003). DOI https://doi.org/10.1016/S0045-7825(02)00603-5. URL http://www.sciencedirect.com/science/article/pii/S0045782502006035

[28] Donea, J., Huerta, A.: Finite Element Methods for Flow Problems. Wiley 500 (2003)

[29] Franca, L., Frey, S.: Stabilized finite element methods: II. the incompressible Navier-Stokes equations. Comput. Meth. Appl. Mech. Engrng. 99, 209-233 (1992)

[30] Franca, L., Russo, A.: Approximation of the Stokes problem by residualfree macro bubbles. East-West J. Numer. Math. 4, 265-278 (1996)

[31] Franca, L.P., Hughes, T.J., Stenberg, R.: Stabilized finite element methods for the Stokes problem. Incompressible computational fluid dynamics pp. 87-107 (1993)

[32] GiD: Personal Pre and Post Processor: URL: http://www.gidhome.com. International Center for Numerical Methods in Engineering (CIMNE) (2010) 
[33] Granzow, B.N., Shephard, M.S., Oberai, A.A.: Output-based error estimation and mesh adaptation for variational multiscale methods. Comput. Meth. Appl. Mech. Engrng. 322, 441-459 (2017)

515 [34] Grätsch, T., Hartmann, F.: Pointwise error estimation and adaptivity for the finite element method using fundamental solutions. Computational Mechanics 37(5), 394-407 (2006)

[35] Guermond, J.: Stabilization of galerkin approximations of transport equations by subgrid modeling. Mathematical Modelling and Numerical Analysis 33(6), 1293-1316 (1999)

[36] Guermond, J., Marra, A., Quartapelle, L.: Subgrid stabilized projection method for $2 \mathrm{~d}$ unsteady flows at high reynolds numbers. Comput. Meth. Appl. Mech. Engrg. 195(6), 5857-5876 (2006)

[37] Hauke, G., Doweidar, M.H., Fuentes, S.: Mesh adaptivity for the transport equation led by variational multiscale error estimators. Int. J. Numer. Meth. Fluids 69, 1835-1850 (2011)

[38] Hauke, G., Doweidar, M.H., Fuster, D.: A posteriori error estimation for computational fluid dynamics. The variational multiscale approach. In: E. Ramm, R. de Borst (eds.) Multiscale Methods in Computational Mechanics, Lecture Notes in Applied and Computational Mechanics, vol. 55. Springer (2010)

[39] Hauke, G., Doweidar, M.H., Miana, M.: The multiscale approach to error estimation and adaptivity. Comput. Meth. Appl. Mech. Engrng. 195, 1573$1593(2006)$

535 [40] Hauke, G., Doweidar, M.H., Miana, M.: Proper intrinsic scales for aposteriori multiscale error estimation. Comput. Meth. Appl. Mech. Engrng 195, 3983-4001 (2006) 
[41] Hauke, G., Fuster, D.: Variational multiscale a-posteriori error estimation for quantities of interest. Journal of Applied Mechanics 76, 021,201(1-6)

540

[42] Hauke, G., Fuster, D., Doweidar, M.H.: Variational multiscale a-posteriori error estimation for the multi-dimensional transport equation. Comput. Meth. Appl. Mech. Engrg. 197, 2701-2718 (2008)

[43] Hauke, G., Fuster, D., Lizarraga, F.: Variational multiscale a posteri-

[47] Hughes, T.: Multiscale phenomena: Green's functions, the Dirichlet-toNeumann formulation, subgrid scale models, bubbles and the origins of stabilized methods. Comput. Meth. Appl. Mech. Engrng. 127, 387-401 (1995)

[48] Hughes, T.: The finite element method:Linear static and dynamic finite element analysis. Dover Publications (2000) 
[49] Hughes, T., Feijoo, G., Mazzei, L., Quincy, J.: The variational multiscale method: A paradigm for computational mechanics. Comput. Meth. Appl. Mech. Engrg. 166, 3-24 (1998)

[50] Hughes, T.J., Franca, L.P.: A new finite element formulation for computational fluid dynamics: VII. the Stokes problem with various well-posed boundary conditions: symmetric formulations that converge for all velocity/pressure spaces. Comput. Meth. Appl. Mech. Engrg. 65(1), 85-96 (1987)

[51] Hughes, T.J., Scovazzi, G., Franca, L.P.: Multiscale and stabilized methods. Encyclopedia of computational mechanics (2017)

[52] Irisarri, D., Hauke, G.: Variational multiscale a posteriori error estimation for 2nd and 4th-order ODEs. International Journal of Numerical Analysis \& Modeling 12(3) (2015)

[53] Irisarri, D., Hauke, G.: A posteriori pointwise error computation for 2-D transport equations based on the variational multiscale method. Computer Methods in Applied Mechanics and Engineering 311, 648-670 (2016)

[54] Irisarri, D., Hauke, G.: Pointwise error estimation for the onedimensional transport equation based on the variational multiscale method. International Journal of Computational Methods 14(4), 30 (2017). DOI 10.1142/S0219876217500402. URL http://www.worldscientific.com/doi/abs/10.1142/S0219876217500402

[55] Irisarri, D., Hauke, G.: A posteriori error estimation and adaptivity based on VMS for the Stokes problem. International Journal for Numerical Methods in Fluids 88(10-11), 493-520 (2018)

[56] Kay, D., Silvester, D.: A posteriori error estimation for stabilized mixed approximations of the Stokes equations. SIAM Journal on Scientific Computing 21(4), 1321-1336 (1999) 
[57] Kechkar, N., Silvester, D.: Analysis of locally stabilized mixed finite element methods for the Stokes problem. Mathematics of Computation 58(197), 1-10 (1992)

595 [58] Larson, M.G., Målqvist, A.: Adaptive variational multiscale methods based on a posteriori error estimation: Duality techniques for elliptic problems. Comput. Science and Engineering 44, 181-193 (2005)

[59] Larson, M.G., Målqvist, A.: Adaptive variational multiscale methods based on a posteriori error estimation: Energy norm estimates for elliptic problems. Comput. Methods Appl. Mech. Engrg. 196, 2313-2324 (2007)

[60] Larsson, F., Díez, P., Huerta, A.: A flux-free a posteriori error estimator for the incompressible Stokes problem using a mixed fe formulation. Computer methods in applied mechanics and engineering 199(37), 2383-2402 (2010)

[61] Masud, A., Khurram, R.: A multiscale/stabilized finite element method for the advection-diffusion equation. Computer Methods in Applied Mechanics and Engineering 193(21), 1997-2018 (2004)

[62] Masud, A., Khurram, R.: A multiscale/stabilized finite element method for the incompressible Navier-Stokes equations. Computer Methods in Applied Mechanics and Engineering 195, 1750-1777 (2006)

[63] Masud, A., Truster, T., Bergman, L.: A variational multiscale a posteriori error estimation method for mixed form of nearly incompressible elasticity. Comput. Methods Appl. Mech. Engrg. 200, 3453-3481 (2011)

[64] Mesri, Y., Bazile, A., Larroya-Huguet, J., Hachem, E.: Parallel and adaptive VMS finite elements formulation for aerothermal problems. Computers \& Fluids 173, 42-50 (2018)

[65] Nobile, F.: A posteriori error estimates for the finite element approximation of the Stokes problem. Tech. rep., ICES, The University of Texas at Austin (2003) 
[66] Oden, J., Prudhomme, S.: New approaches to error estimation and adaptivity for the Stokes and Oseen equations. International journal for numerical methods in fluids 31(1), 3-15 (1999)

[67] Oden, J.T., Wu, W., Ainsworth, M.: An a posteriori error estimate for finite element approximations of the Navier-Stokes equations. Computer Methods in Applied Mechanics and Engineering 111(1-2), 185-202 (1994)

[68] Oñate, E., Arteaga, J., Garcia, J., Flores, R.: Error estimation and mesh adaptivity in incompressible viscous flows using a residual power approach. Comput. Methods Appl. Mech. Engrg. 195, 339-362 (2006)

[69] Parés, N., Díez, P., Huerta, A.: Subdomain-based flux-free a posteriori error estimators. Comput. Methods Appl. Mech. Engrng. 195, 297-323 (2006)

[70] Pierre, R.: Simple C0 approximations for the computation of incompressible flows. Computer Methods in Applied Mechanics and Engineering 68(2), 205-227 (1988)

[71] Rasthofer, U., Gravemeier, V.: Recent developments in variational multiscale methods for large-eddy simulation of turbulent flow. Arch Computat Methods Eng 25, 647-690 (2018)

[72] Rebollo, T.C., Fernandez-Garcia, S.: On the computation of the stabilized coefficients for the 1D spectral VMS method. SEMA 75, 573-590 (2018)

[73] Rossi, R., Cotela, J., Lafontaine, N., Dadvand, P., Idelsohn, S.: Parallel adaptive mesh refinement for incompressible flow problems. Computers \& Fluids 80, 342-355 (2013)

[74] Russo, A.: A posteriori error estimators for the Stokes problem. Applied Mathematics Letters 8(2), 1-4 (1995)

[75] Song, L., Hou, Y., Zheng, H.: Adaptive variational multiscale method for the Stokes equations. International Journal for Numerical Methods in Fluids 71(11), 1369-1381 (2013) 
[76] Verfürth, R.: A posteriori error estimators for the Stokes problem. Numer. Math. 55, 309-325 (1989)

[77] Zheng, H., Hou, Y., Shi, F.: Adaptive variational multiscale methods for 650 incompressible flow based on two local gauss integrations. Journal of Computational Physics 229(19), 7030-7041 (2010) 